\title{
Making Arm Movements Within Different Parts of Space: Dynamic Aspects in the Primate Motor Cortex
}

\author{
R. Caminiti, ${ }^{1}$ P. B. Johnson, ${ }^{2}$ and A. Urbano ${ }^{1}$ \\ "Istituto di Fisiologia umana, Facoltà di Medicina e Chirurgia, Università degli Studi di Roma "La Sapienza," 00185 Rome, \\ Italy, and ${ }^{2}$ Curriculum in Neurobiology, University of North Carolina, Chapel Hill, North Carolina 27599, USA
}

The activity of 176 individual cells in the arm area of motor cortex (areas 4 and 6 ) was studied while monkeys made arm movements of similar direction within different parts of extrapersonal space. The behavioral paradigm used was a 3-dimensional reaction-time task aimed at dissociating the direction of movement, which remained similar across the work space, from the patterns of muscular activity and the angular joint excursions necessary to perform these movements.

In agreement with other studies (Georgopoulos et al., 1982; Schwartz et al., 1988), we found that, within a given part of space, the activity of $169(96.0 \%)$ cells studied increased most for a given preferred direction and less for other directions of movement. This change was graded in an orderly fashion. We further analyzed the orientation in space of the cells' preferred directions under the differing conditions of the task. We found that, as movements with similar trajectories were made within different parts of space, the cells' preferred directions changed spatial orientation. This change was of different magnitudes for different cells, but at the level of the population, it followed closely the changes in orientation of the arm necessary to perform the movements required by the task.

Movement population vectors (Georgopoulos et al., 1983, $1986,1988)$ computed from cell activity proved to be good predictors of movement direction regardless of where in space the movements were performed.

These results indicate that motor cortical cells can code direction of movement in a way which is dependent on the position of the arm in space. The data are discussed in relation to the existence of mechanisms which facilitate the transformation between extrinsic and intrinsic coordinates. These transformations are necessary to perform arm movements to visual targets in space.

Movement can be represented in many different domains, relating to dynamic, kinematic, and other aspects of motor be-

\footnotetext{
Received July 25, 1989; revised Nov. 13, 1989; accepted Dec. 21, 1989.

This work was supported by The European Economic Community, the Consiglio Nazionale delle Ricerche, Rome, Italy, and USPHS Grant NSO7166. We are grateful to P. Battistoni and G. Motta for providing graphic software and to Prof. M. Salerno for the use of the graphic facilities at the Department of Electronic Engineering, University of Tor Vergata, Rome. We are especially thankful to Yves Burnod for his many useful ideas and suggestions concerning this study, to $\mathrm{C}$. Galli for his assistance in the preparation of the manuscript, and to E. Macellari for the design of the laboratory interfaces.

Correspondence should be addressed to Roberto Caminiti, Istituto di Fisiologia umana, Facoltà di Medicina e Chirurgia, Università di Roma "La Sapienza," Piazzale A. Moro 5, 00185 Rome, Italy.
}

Copyright (C) 1990 Society for Neuroscience $0270-6474 / 90 / 072039-20 \$ 03.00 / 0$ havior (Saltzman, 1979; Stein, 1982). Studies of some forms of multiarticular motion in space, such as reaching to an object of interest, have been aimed at uncovering patterns of invariance in the underlying motor variables on the assumption that such regularities would have revealed the parameters coded by the CNS. For example, the observation that in many instances the hand follows a roughly straight-line or mildly curved path when moving between pairs of points in space (Gilman et al., 1976; Morasso, 1981; Abend et al., 1982; Flash and Hogan, 1985; Hollerbach and Atkeson, 1987) has led to the proposition that hand trajectory is one of the movement variables encoded (Morasso, 1981; Hogan, 1984; Flash and Hogan, 1985; see Hogan, 1988 , for a discussion) and that this encoding occurs within an extracorporeal Cartesian coordinate system. Constant relationships between joint angular velocities during arm movements (Soechting and Lacquaniti, 1981; Lacquaniti and Soechting, 1982) have suggested, instead, an encoding mechanism using joint variables, and therefore occurring within a preferred intrinsic frame of reference (see Soechting and Terzuolo, 1988, for a discussion).

The mechanisms by which the coordinate systems used for the planning and execution of arm movements are represented in the cerebral cortex are not known. Many neurophysiological studies of motor cortical areas have been devoted to the analysis of the relationships between neural activity and movement variables. Significant relationships were first shown between the activity of motor cortical cells and the force exerted by alert behaving monkeys (Evarts, 1968, 1969; Humphrey et al., 1970; Schmidt et al., 1975; Smith et al., 1975; Hepp-Reymond et al., 1978; Thach, 1978; Cheney and Fetz, 1980; Evarts et al., 1983). Attention has also been devoted to the correlation between motor cortical activity and direction (Humphrey et al. 1970; Schmidt et al., 1975; Thach, 1978) and velocity (Humphrey et al., 1970; Hamada and Kubota, 1979) of movement. These works, of seminal importance in the study of the movement variables encoded in the activity of motor cortical populations, have not provided detailed information about the frame of reference used by the cerebral cortex to represent arm movement direction in space. This has been, in part, a consequence of the methodological choice to reduce the number of degrees of freedom of movement in order to facilitate the quantitative evaluation of the correlation between behavior and neural activity. The movements tested were, in most instances, motions of a single joint. It has since been shown that multijoint movements are accomplished by dynamic interactions among limb segments (Hollerbach and Flash, 1982) which are not present in single-joint movements. While single-joint movements are mainly based on the relations of an agonist-antagonist pair, multijoint movements require the synergistic action of several muscles. 

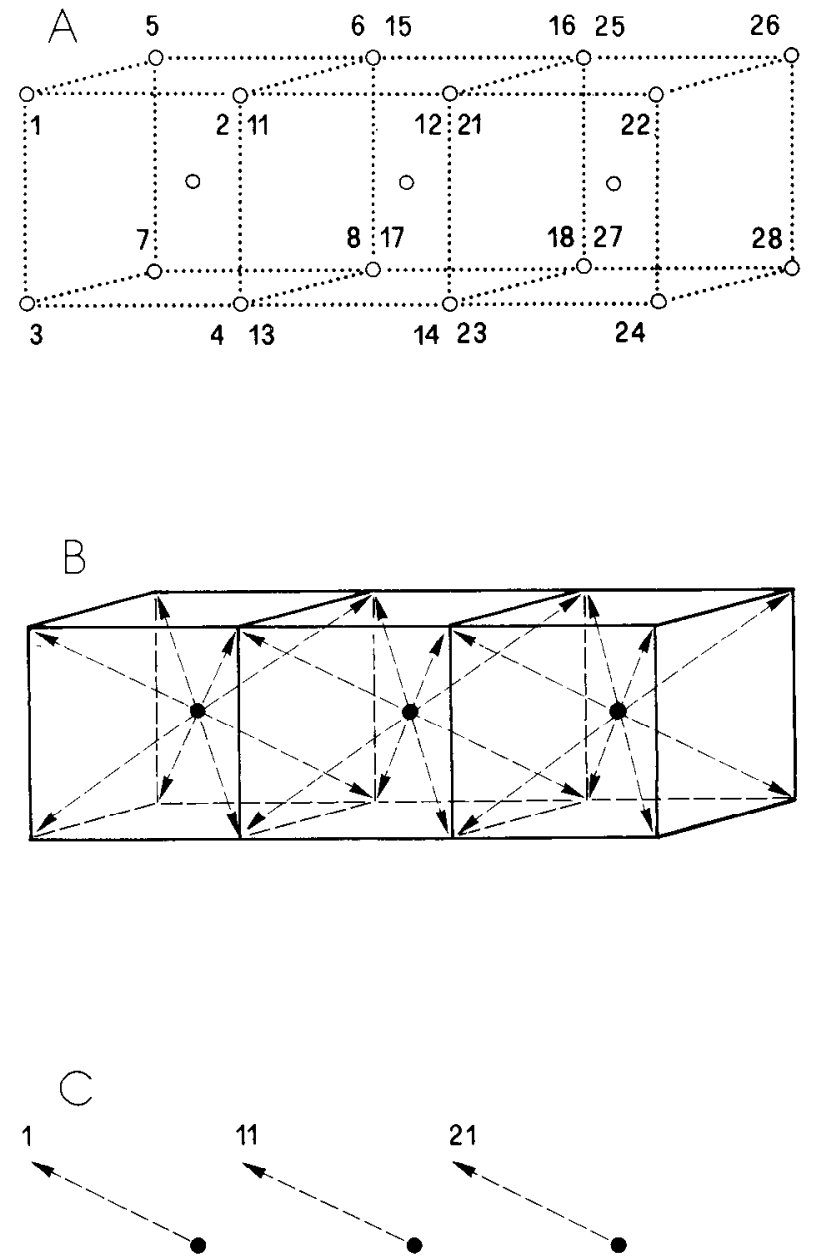

Figure 1. Apparatus and task. A, Layout of the work space with pushbuttons (target lights) indicated by open circles. Numbers identify target position in space. Some pushbuttons are labeled by 2 numbers $(2,11$; 12,21 ; etc.), indicating that in the task they are targets of movements of 2 different origins. Numbers also identify the direction of movement. Thus, 2 and 11 identify movements starting from the centers of the left and central parts of the work space, respectively, and directed to target light $(2,11)$. The animal was seated on a primate chair, $25 \mathrm{~cm}$ away from the front lights. The center of the central cube was aligned with the body midline at shoulder height (see also Fig. 2). B, Layout of the task showing 3 sets of movement directions performed in the left, center, and right parts of the work space. Filled circles indicate movement origins within each part of space where monkeys made equal-amplitude $(8.7 \mathrm{~cm}$ ) movements with the same origin in 8 different directions (arrows). $C, 1,11,21$ indicate one of the 8 triplets of movements which traveled along parallel paths in different parts of the work space.

Subsequent studies in which 2-dimensional arm movements aimed at visual targets have been accurately monitored (Georgopoulos et al., 1982) revealed the existence of a significant correlation between direction of movement and neural activity in both motor (Georgopoulos et al., 1982) and posterior parietal (Kalaska et al., 1983) cortices. This relation was finely graded: cell activity was highest with movement in a "preferred direction" and decreased in an orderly way for other movement directions. The relatively broad tuning of these cells has led to the proposition that direction of movement is represented by a population rather than a single cell code (Georgopoulos et al., 1983). Recent studies have extended these observations to movements occurring in a 3-dimensional domain (Georgopou- los et al., 1988; Schwartz et al., 1988) and have elucidated the interaction between direction of movement and direction of force as a determinant of motor cortical cell activity (Kalaska et al., 1989).

These studies using free-arm movements provide a background for addressing the question of which coordinate systems are used by motor cortical areas to represent movement direction in space. Direction of movement can, in fact, be coded within a preferred extrinsic extrapersonal coordinate system or within an intrinsic corporeal frame of reference, such as patterns of muscular activity or joint variables. A third interesting possibility is that information from both of these domains of representation are combined to code direction of movement. These alternatives can be tested experimentally assuming that a salient property of motor cortical cells is their directionality. The directionality of each cell can be represented as a vector of a given orientation in the 3-dimensional space. If these vectors are represented in an extrapersonal Cartesian space, then the preferred directions of motor cortical cells involved in the generation of different arm trajectories should not change when similar trajectories are repeated within different parts of space and therefore using different patterns of muscle activity and different angular excursions of the shoulder joint. On the contrary, an orderly shift of the orientation in the space of the cells preferred directions would be suggestive of a coding of arm movement direction within a frame of reference that can be preferentially intrinsic (body centered) or able to combine extrinsic and intrinsic information.

To address these questions, we recorded the activity of individual neurons in the motor cortex of monkeys while they made arm movements of similar direction within different parts of extrapersonal space. The task was aimed at achieving a dissociation between movement direction and underlying patterns of muscle activity and between intrinsic corporeal frames of reference and extrinsic ones.

\section{Materials and Methods}

Apparatus and task. The behavioral apparatus consisted of 19 metal rods placed in front of and oriented towards the monkey. The end of each rod was fitted with a 1-cm-diameter transparent plastic push button which could be illuminated from behind by a red light-emitting diode (LED). Sixteen of the push buttons were arranged in space so as to form the vertices of 3 adjacent imaginary cubes ( 8 buttons being employed as vertices of 2 contiguous cubes; Fig. $1 A$ ). The 3 remaining buttons were placed at the centers of these 3 cubes. The buttons at the vertices of each cube were placed $10 \mathrm{~cm}$ from one another and $8.7 \mathrm{~cm}$ from the center button of that cube. The center button of the center cube was located in the midsagittal plane at shoulder height and $25 \mathrm{~cm}$ from the animal (Fig. 2). The animal sat in a primate chair and made free arm movements at visual targets in a 3-dimensional (3-D) space with limited motion of the torso.

Three adult female Macaca nemestrina monkeys (3-4 kg) were trained to move their hands in a visual reaction-time task. After a variable intertrial interval $(1-1.5 \mathrm{sec})$, a trial was initiated by the illumination of the center button of the central cube. The monkey was required to press and hold the center button for a randomly variable period of time (control time, CT; $1.5-2.5 \mathrm{sec}$ ) until the center LED was turned off and a button at one of the vertices of the central cube was illuminated. Within specified upper limits of reaction time (RT; $350 \mathrm{msec}$ ) and movement time (MT; $1 \mathrm{sec}$ ), the animal was required to move its hand to the target button and depress it for a variable period of time (target holding time, THT; $1-1.5 \mathrm{sec}$ ), in order to receive a liquid reward. This sequence was repeated until 5 movements to each of 8 targets were correctly performed. The targets were presented in a pseudorandom fashion (Cochran and Cox, 1957) so that the animal could not predict when or where the next target would appear. Movements were then repeated on the left and right cubes. The sequence of cube presentation 


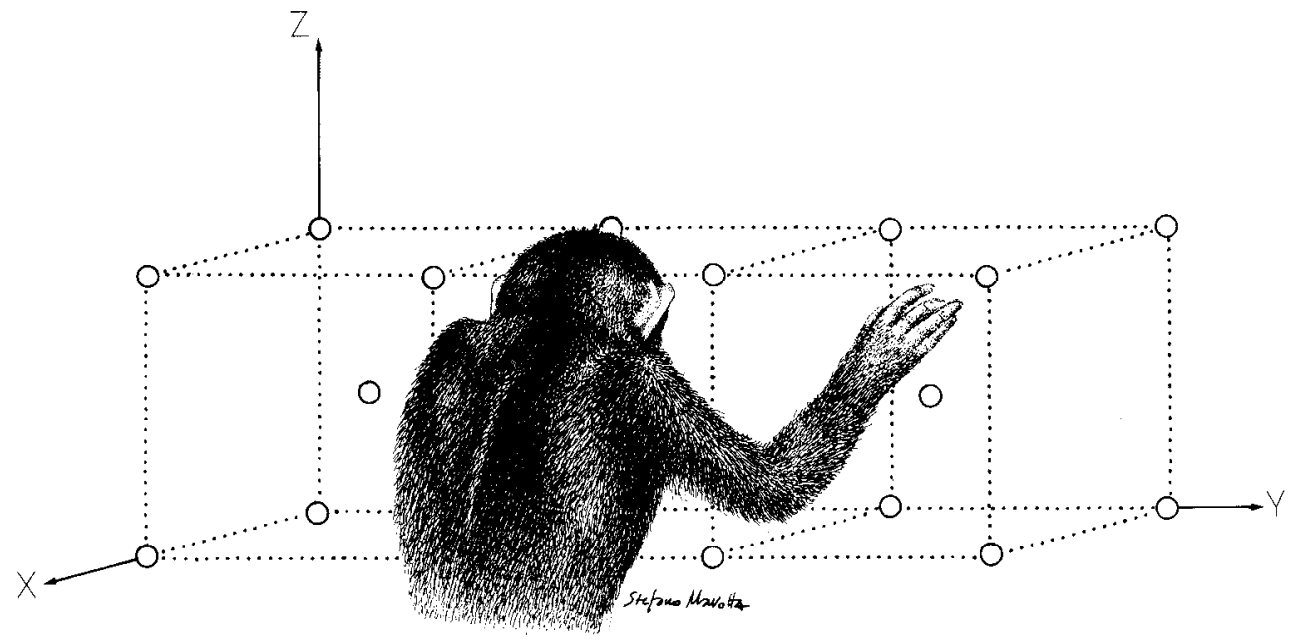

Figure 2. Layout of the task relative to the $X, Y, Z$ spatial coordinates and to the animal's body. was often changed. Thus, within any one cube, the animals made arm movements in 8 different directions, all starting from a common central position (Fig. $1 B$ ). Because of the side-by-side arrangement of the cubes, the 8 different directions in any 1 cube were parallel to the 8 different movement directions in both of the other cubes but occurred within different regions of extrapersonal space (Fig. 1C). Throughout this paper, the term "triplet" will be used to refer to a group of 3 such parallel movements. The collection of data from each individual neuron lasted at least $20 \mathrm{~min}$. To exclude possible changes in cell activity over time, on some occasions the data collected during the initial cube presentation were recollected.

Recording of movement trajectories. The movements of the arm were recorded using a sonic tracking system (Science Accessories Corporation, GP-8-3D). A spark gap was mounted on the radial aspect of the monkey's wrist. The ultrasonic signal emitted by the spark gap was detected by at least 3 of 4 microphones mounted on the roof of the recording room. The $X Y Z$ coordinates of the sparker were calculated from the signal delays at a rate of 35 samples/sec. Recording of movement trajectories and neural recordings were performed in separate sessions.

Electromyographic (EMG) recordings. EMG activity was recorded by means of Teflon-coated multistranded stainless stecl clectrodes, inserted intramuscularly. The raw EMG signals were rectified and filtered. The signal was then recorded digitally at a sampling frequency of $100 \mathrm{~Hz}$. EMG and neural recording sessions were performed separately. The activities of the following muscles (Howell and Strauss, 1933) were recorded during the task: spinodeltoid, clavicular deltoid, cranial trapezius, caudal trapezius, triceps (lateral head), triceps (long head), biceps longus, teres major, and pectoralis. Since it is reasonable to suppose that EMG activity occurring shortly before the hand reached the target was not related to the generation, but more likely to the braking of movement, for subsequent quantitative analyses, we considered only the EMG activity recorded from target presentation to $200 \mathrm{msec}$ after movement onset.

Neural recordings. At the end of training, the animals were anesthetized with sodium pentobarbital (Ncmbutal, $40 \mathrm{mg} / \mathrm{kg}$, i.p.) and a $20-\mathrm{mm}$ diameter hole was made in the skull over the motor cortex. A cylindrical recording chamber was then cemented in place using sterile dental acrylic. A threaded metal bar was also cemented to the skull to allow the head to be fixed during neural recording. When not being recorded from, the chamber was filled with sterile saline solution and closed with a plug. At the beginning of each recording session, the plug was removed, and a Chubbuck microdrive (see Mountcastle et al., 1975) was mounted on the chamber, which was then filled with mineral oil. The microelectrodes were platinum/iridium alloy wires, etched to obtain 1- to $2-\mu \mathrm{m}$ wide tips and then insulated with glass. Tip impedance during recording was generally $1-3 \mathrm{M} \Omega$. The electrodes were lowered into the brain through an intact dura. One or 2 penetrations were made during each recording session. Loci of microelectrode penetrations were marked on a grid map of the chamber. In each hemisphere, recording was continued for approximately $30 \mathrm{~d}$. The electrical activity of individual neurons was recorded extracellularly. Only initially negative action potentials were studied. The signals of single neurons were isolated using a time-amplitude window discriminator, recorded in the form of interspike intervals with $0.1 \mathrm{msec}$ resolution, displayed on-line as rasters of spikes, and stored in digital form. The depth at which each cell was studied along a penetration was marked on protocols and was expressed relative to the first cell encountered in the cortex and to the boundary between cortex and white matter, as judged from the absence of initially negative spikes in the record. On some occasions, small electrolytic lesions were made to facilitate the histological reconstruction of the penetration. When performed, a $3-\mu \mathrm{A}$ current was passed for $3 \mathrm{sec}$ through the tip of the electrode. A detailed qualitative examination of each cell encountered was aimed at assessing whether neural activity was related to arm movements at the shoulder and/or elbow joints and to determine the presence and quality of passive inputs to the cell from skin, deep tissues, muscles, and joints. The aim of the qualitative examination was to select, for the quantitative analysis, only those cells which modulated their activity with proximal arm movements. Since cells related to wrist or digit movements are engaged late during reaching (Murphy et al., 1985) and probably do not participate directly in the generation of the types of movements performed in the task, they were not further analyzed. Recordings were made in the hemisphere contralateral to the arm used to perform the task. Data from the right hemisphere were mirrortransformed to allow the analysis as if they were recorded from the left hemisphere (right arm).

Dala acquisition. Control of the behavioral task and collection of all data were performed by a PDP $11 / 23+$ minicomputer. The computer presented target lights, monitored the status of the push buttons, and recorded neuronal and $E M G$ data. Any trials in which the monkey did not perform the behavioral task correctly were rejected on-line. Data were displayed as they were acquired to allow the experimenter to reject recorded artifacts.

Quantitative data analysis. For the purposes of data analysis, each trial was divided into 4 epochs. The CT was the last $1 \mathrm{sec}$ that the animal held the center button prior to the presentation of the target light. RT was the time from the presentation of the target to the release of the center button. The MT was defined as the time from the release of the center button until the target button was first pressed. Finally, the THT began when the target button was pressed and ended with the presentation of the reward. In addition, a fifth epoch, the total experimental time (TET), was defined as the combination of the RT and MT.

For every recorded neuron, the mean firing rate within the TET of each trial was calculated. A standard analysis of variance (ANOVA) was performed on these rates. A cell whose discharge rate during the TET varied significantly $(p<0.05)$ with different movement directions within a cube was considered directional in that cube. Only those cells that were directional in more than 1 cube were subjected to further analysis in this study.

To assess whether cell activity changed when movements were made in different parts of space a 2-factor ANOVA was performed on the changes in firing frequency from the CT. The 2 factors in the ANOVA model were the cube (the spatial region where the animal performed) and direction of movement. For this analysis, parallel movement di- 
rections (i.e., 1-11-21, etc.) within different parts of space were considered as a unique similar movement direction. The space-direction interaction term of this analysis was used as an indicator of the existence of significant changes in the directional properties of cells when movements were made in different parts of space. The same analysis and criteria were adopted to evaluate changes in muscle activity in the different conditions of the task.

To further quantify the relationship between direction of movement and frequency of neuronal discharge, a multiple-regression analysis was performed. For each cell, in each cube in which it was directional, the firing frequency was linearly regressed on the direction of movement using a least-squares method (Draper and Smith, 1981). The following regression model was used for each cell-cube combination:

$$
\hat{f}=b_{0}+b_{x} m_{x}+b_{y} m_{y}+b_{z} m_{z} \text {, }
$$

where $\hat{f}$ is the predicted frequency of discharge of the cell, $b_{0}-b_{z}$ are the coefficients determined by the regression procedure, and $m_{x}, m_{y}$, and $m_{z}$ are the $X, Y$, and $Z$ components of the direction of arm movement. The movement directions were defined as the vector difference between the final and initial hand positions. The orientations of the axes with respect to the animal and the behavioral apparatus are shown in Figure 2 . The regression $F$-test was used to identify those cells which fit the model. Cells with significant regressions $(p<0.05)$ were considered to be directionally tuned.

Analysis of preferred direction and spherical regression. Regression equation (1) predicts that there is a particular movement direction for which the cell discharge frequency will be maximal. This is the cell's preferred direction, and it can be represented by the preferred direction vector $C$, the components of which are calculated from the regression coefficients $b_{x}, b_{y}$ and $b_{z}$ (Schwartz et al., 1988) :

$$
c_{x}=b_{x} / r, \quad c_{y}=b_{y} / r, \quad c_{z}=b_{z} / r,
$$

where

$$
r-\left(b_{x}^{2}+b_{y}{ }^{2}+b_{z}^{2}\right)^{1 / 2} .
$$

A preferred direction for each cell was calculated for every cube in which that cell was directionally tuned. Thus, for each neuron, up to 3 preferred directions were determined as the monkey made movements in the left, center, and right regions of extrapersonal space. The distributions of these preferred direction vectors were examined by generating 3-D plots and 2-D equal-area projection plots (Watson, 1983) for the vectors obtained from the left, center, and right cubes. A qualitative indication of the variation of the preferred direction vectors across space was obtained by selecting cells which fell in particular sectors of the equal-area projection plot of the central cube and by "tracking" their locations in the left and right cubes.

To quantify further any changes in a particular cell's preferred direction vector as the monkey worked in various parts of space, spherical angular differences were calculated and plotted. Between any 2 preferred direction vectors of any 1 cell, the spherical angular difference, $S$, was defined as:

$$
S=\cos ^{-1}\left(c 1_{x} c 2_{x}+c 1_{y} c 2_{y}+c 1_{z} c 2_{z}\right),
$$

where $c 1_{x}, c 1_{y}$, and $c 1_{z}$ are the components of 1 vector and $c 2_{x}, c 2_{y}$, and $c 2_{z}$ are the components of the second vector.

As a measure of whether the observed changes in the preferred directions of individual cells was consistent across the entire population, the spherical correlation coefficient of Fisher and Lee (1983) was calculated. Significance levels for the correlation coefficient were calculated using statistical bootstrapping techniques (Efron, 1979). A significant correlation permits the computation of the spherical regression (Jupp and Mardia, 1980) to determine the manner in which the populations of vectors are correlated. The spherical regression uses a least-squares method to determine the elements of $R$ in the following model:

$$
\hat{a}_{i}=(\mathbf{R}) b_{i},
$$

where $b_{i}$ is the $i^{\text {in }}$ cell's preferred direction vector in 1 cube, $\hat{a}_{i}$ is the predicted preferred dircetion vector of the same cell in another cube, and $\mathbf{R}$ is a $3 \times 3$ rotation matrix. The regression coefficients (the elements of matrix $\mathbf{R}$ ) determine the angles through which the population of preferred direction vectors in 1 part of space must be rotated to result in maximal alignment of those vectors with their corresponding vectors in another part of space. The matrix $\mathbf{R}$ was calculated as

$$
\mathbf{R}=\left(\mathbf{D}^{\prime} \mathbf{D}\right)^{-1 / 2} \mathbf{D}^{\prime},
$$

where the symmetric positive-definite square root is taken, $D$ is the expected value of $a b^{\prime}$, and $\mathbf{D}^{\prime}$ and $b^{\prime}$ are the transposes of $\mathbf{D}$ and $b$, respectively. That is,

$$
\mathbf{D}=\frac{1}{N} \sum_{i=1}^{N}\left(a_{i} b_{i}\right)
$$

where $N$ is the number of cells included in the regression. Spherical regressions were performed between preferred directions calculated in the left and center, center and right, and left and right parts of space. In any particular regression, only those cells that were directionally tuned in both of the relevant cubes were included.

The rotation angles $\psi, \theta$, and $\Phi$, about the $X, Y$, and $Z$ axes, respectively, were computed from the rotation matrix, $\mathbf{R}$, using standard equations of rotation. The positive direction of rotation about the $Z$ axis was defined as a clockwise rotation (viewed from above). Similarly, positive rotation about the $X$ axis was defined as clockwise when viewed from the right, and positive rotation about the $Y$ axis was defined as clockwise when viewed from behind the animal. Since, in the above equations, the $a_{i}$ vectors always came from the right-most and the $b_{i}$ vectors from the left-most of the 2 cubes, the calculated angles indicate the rotation necessary to predict the vectors of the right-most region of the work space from the vectors of the left-most region. The order of the rotations about the $X, Y$, and $Z$ axes is determined by the method used to calculate these from the rotation matrix. Due to the fact that the second and third rotations are around the axes which have already been modified by the previous rotations, the order of rotation can influence the values of the resulting angles. Various orders of rotation were performed to ascertain the magnitude of this effect. Confidence intervals for the angles of rotation were calculated using statistical bootstrapping techniques (Efron, 1979).

To determine the rotation about the $Z$ axis of the preferred directions of individual cells, these preferred directions were projected into the horizontal plane. Within this plane, the angular differences between vectors of the different parts of the work space were calculated. As with the spherical regression, positive angles represent a clockwise rotation of the preferred direction (when viewed from above) as the monkey moved its arm from left to right. Frequency histograms of these angular differences were then plotted.

Analysis of population vectors. Population vectors (Georgopoulos et al., 1983, 1986, 1988) were computed for all movement directions in each of the 3 regions of space where the animals performed the task. A population vector, for a given direction of movement, is the sum of the vectorial contributions made by the preferred directions of a population of motor cortical cells. Different weighting functions have been proposed (Georgopoulos et al., 1988) to calculate population vectors. We adopted weighting function 2 from Georgopoulos et al. (1988).

Histological data analysis. At the end of recording, the animals werc administered a lethal dose of Nembutal. The dura was removed and metal pins (usually 4) were inserted into the cortex using a template which mimicked the base of the microdrive. This allowed positioning of the pins at known coordinates within the chamber. Pin coordinates were chosen so as to bound the area in which the penetrations were performed. These were later related to macroscopic features of the brain and, after histological analysis, to architectonic fields. The brain was. then removed, fixed in buffered formalin and allowed to sink in a $30 \%$ sucrose solution. Finally, it was cut on a microtome in a plane parallel to that defined by the insertion of the pins. Sections ( $40 \mu \mathrm{m}$ thick) were stained with $1 \%$ toluidine blue.

\section{Results}

\section{Movement trajectories}

The task employed in this study was aimed at maintaining similar direction of movement across the work space while changing the underlying patterns of muscular activity and joint angles. Figures 3 and 4 show examples of hand trajectories recorded during the performance of the task. In most instances, the path followed by the hand described a curved trajectory in space. No gross irregularities were observed in these trajectories regardless of where in space the movements were performed. It can be seen that trajectories of different replications of the same movement direction were highly stereotyped. This level of per- 

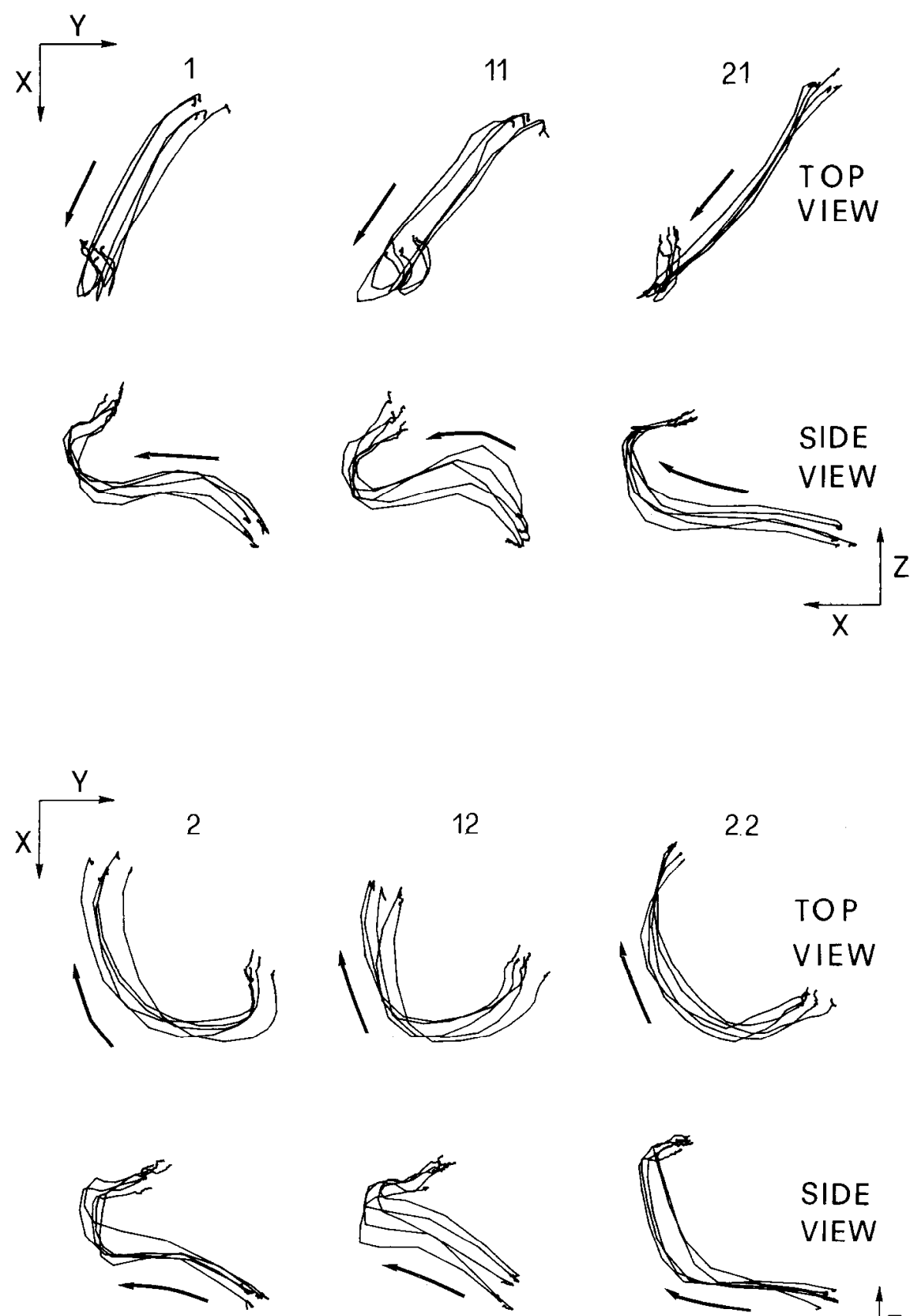
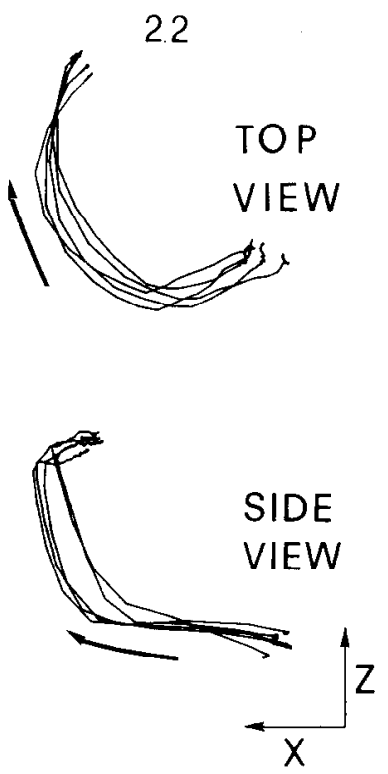

Figure 3. Two-dimensional plots of top and side views of hand trajectories for movements in directions $1-11-21$ and 2-12-22. Side views are taken from the right. Five replications are shown for each movement direction. Arrows indicate movement direction. $X, Y$, and $Z$ axes correspond to those shown in Figure 2. Axis calibration bars, $2 \mathrm{~cm}$. Movements were performed with the left arm. formance was a result of intensive training which was continued until a criterion level of $90 \%$ correct trials was reached. This was generally achieved in 30-45 d of training, depending on the animal.

Figure 3 shows top and side views of trajectories in movement directions 1-11-21 and 2-12-22. It can be seen that movements made within different parts of the work space traveled along paths which were highly similar and in some cases almost parallel. Similar results were obtained for triplets 3-13-23 and 4-1424 (Fig. 4), as well as for the remaining triplets of movement directions.

\section{EMG analysis}

There were 2 main reasons for the EMG analysis, namely, to check whether both individual muscles and the pattern of aclivity of all muscles involved in the task varied as the animals made movements of similar direction across the work space. The results of this analysis are documented in Figures 5 and 6 , where the activity of each individual muscle is shown for each of the 8 triplets of movement directions studied in the task. Within a given part of space, as shown by previous studies (Massey et al., 1981; Georgopoulos et al., 1984a), EMG activity 

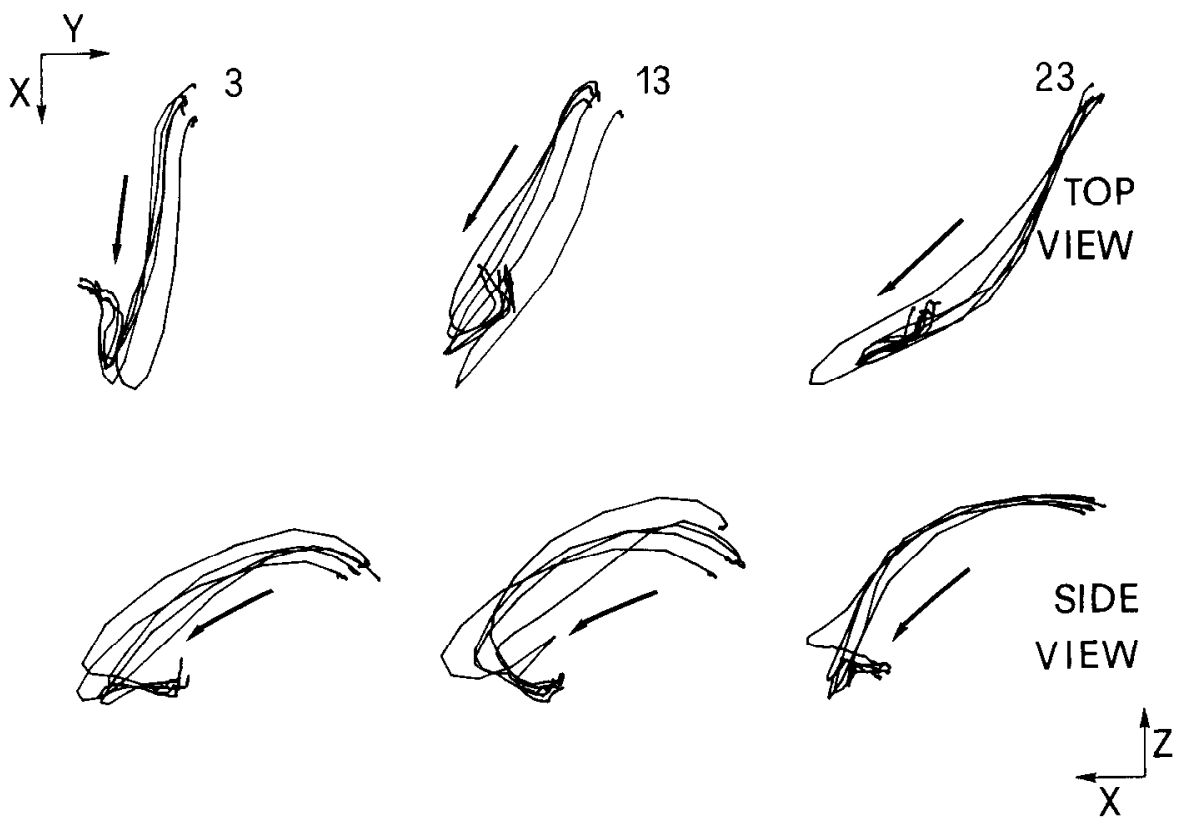

$x \stackrel{Y}{\longrightarrow}$
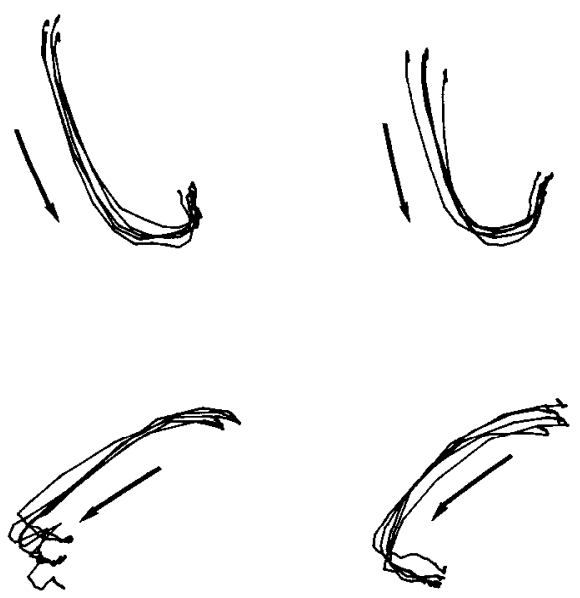

14
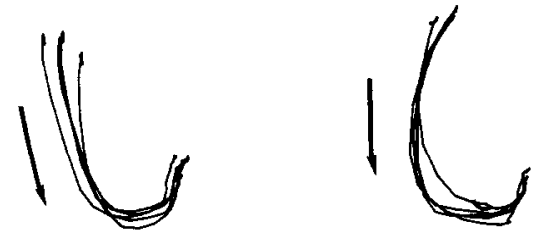

24

TOP

VIEW
Figure 4. Two-dimensional plots of top and side views of hand trajectories for movements in directions 3-13-23 and 4-14-24. Conventions and symbols as in Figure 3. varied in an orderly fashion with direction of movement. More important for our purposes is that, in addition, all aspects of EMG activity (intensity, sign of modulation, and temporal relations to movement onset) varied as movements of similar directions were made in different parts of the work space. Consider, for instance, the activity of the caudal trapezius (Figs. 5, 6 ) around the movement onset. The initial burst, visible for movement direction 2 , decreased in amplitude for movement direction 12 and was even more reduced in direction 22 . The activity of this muscle changed significantly in all the triplets of this task, excluding 1-11-21. Similar observations were made for most of the muscles studied. Significant space-direction interactions (ANOVA, $F$-test, $p<0.05$ ) were found for the following muscles: lateral head of triceps, caudal trapezius, spinal

Figure 5. EMG activity of 9 proximal muscles recorded during the task. Activity is shown for triplets (1-11-21, 2-12-22, 3-13-23, 4-14-24) of movements having similar directions but performed within different parts of space. In each panel, from top to bottom, data refer to the activity of caudal trapezius, cranial trapezius, spinal deltoid, clavicular deltoid, long head of triceps, lateral head of triceps, biceps longus, teres major, and pectoralis. Numbers on the horizontal axis represent milliseconds relative to the onset of movement (0). Data represent averages of 5 trials and are aligned to movement onset. Movements were performed with the left arm. 


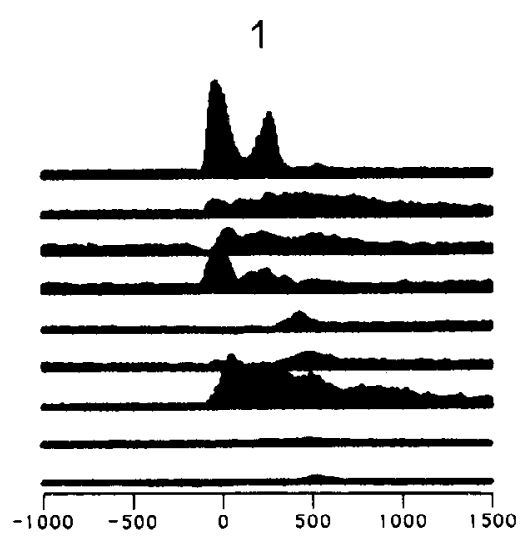

2

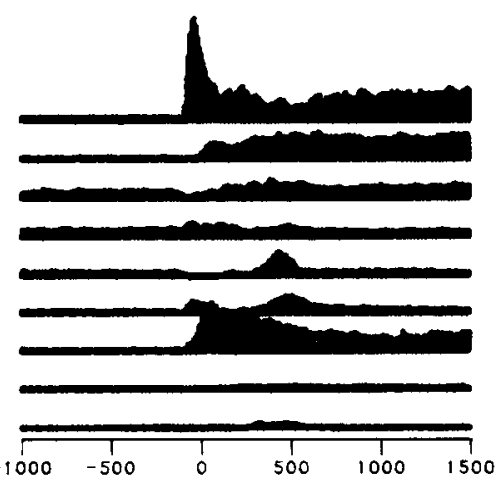

3

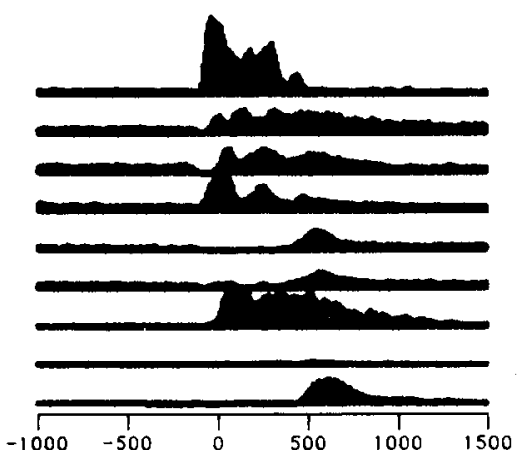

4

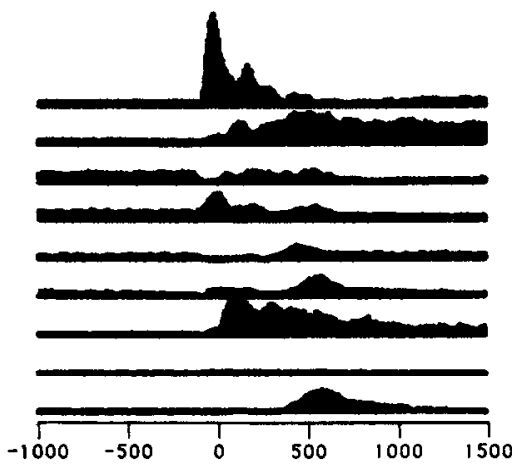

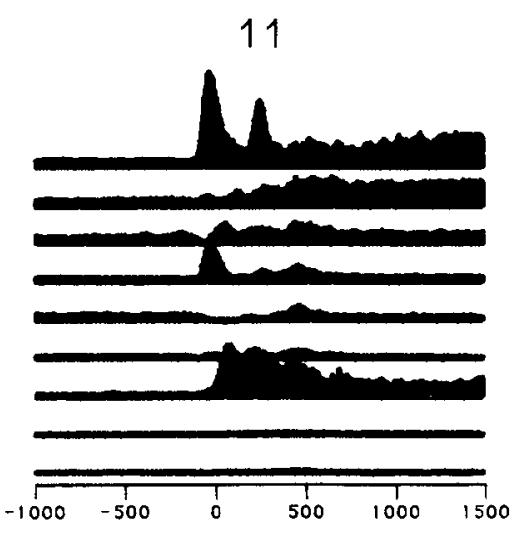

12

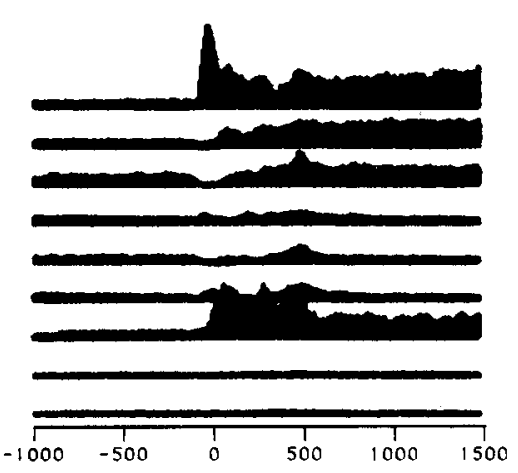

13

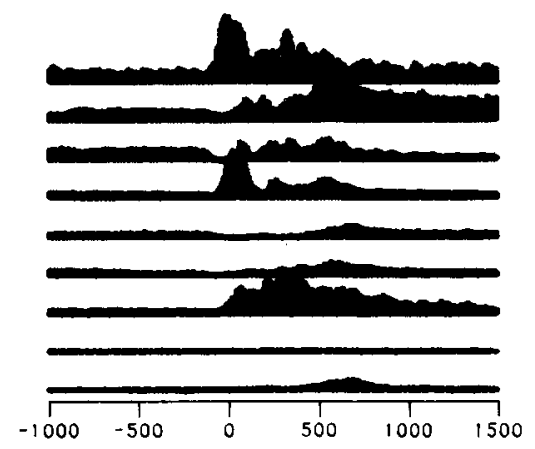

14

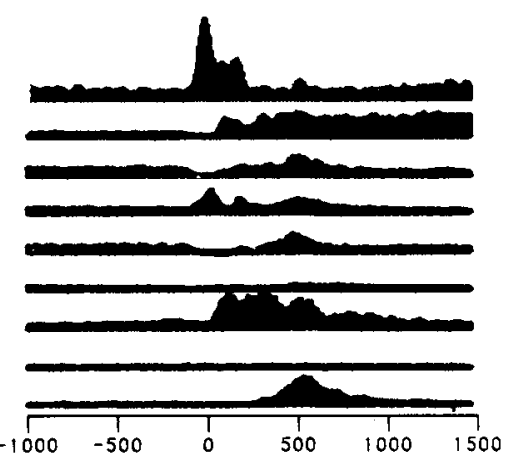

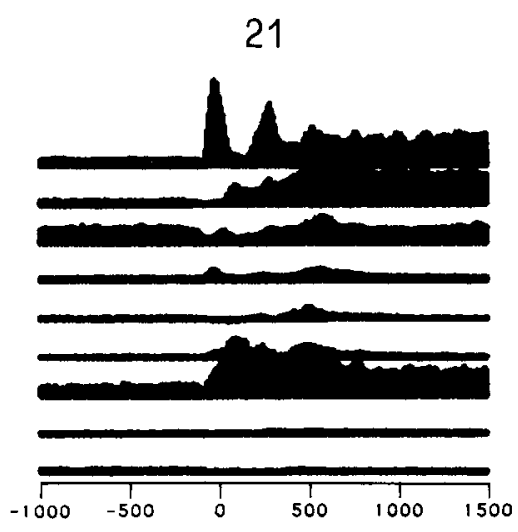

22

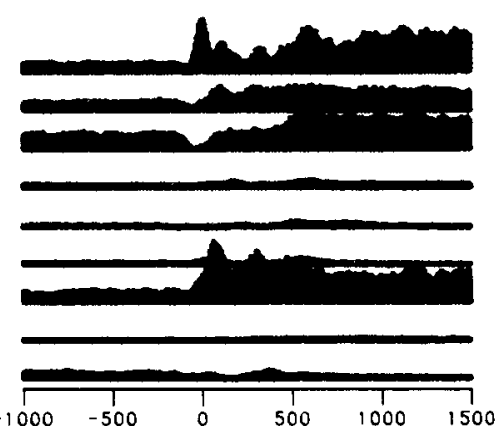

23

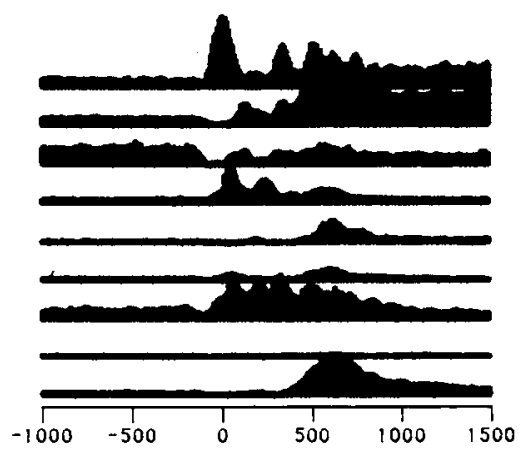

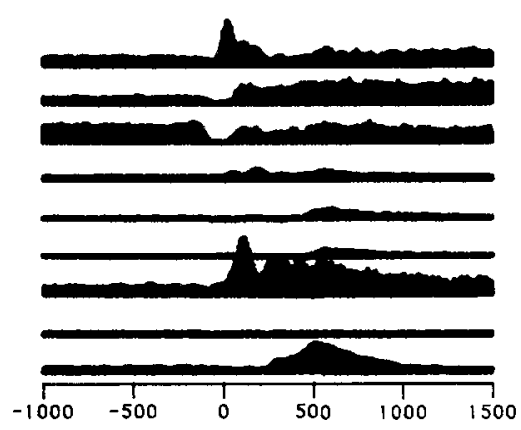




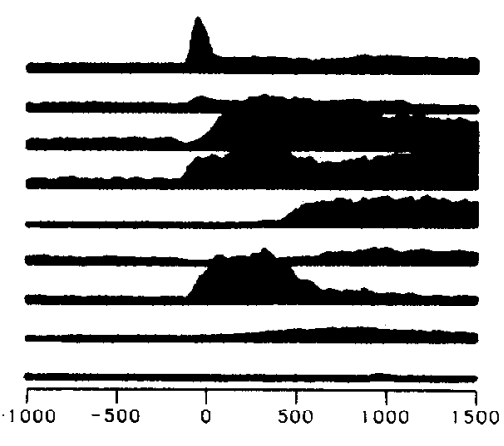

6

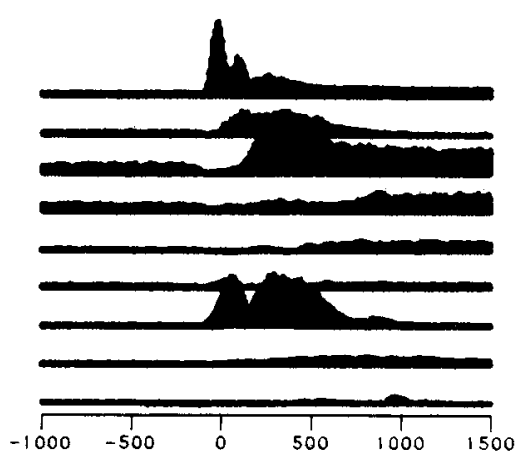

7

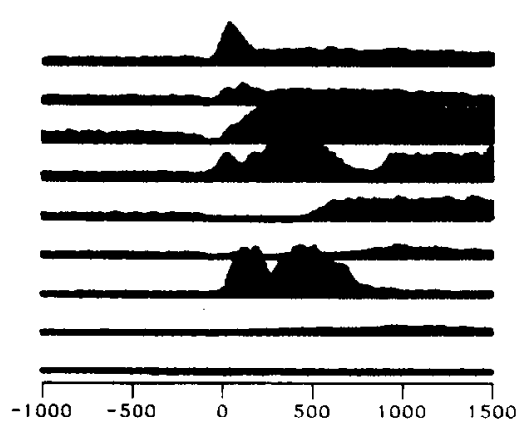

8

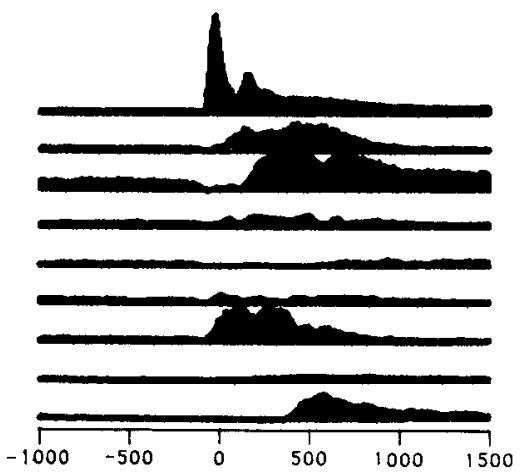

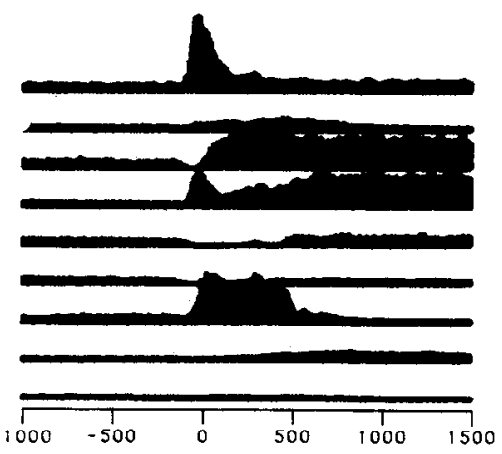

16

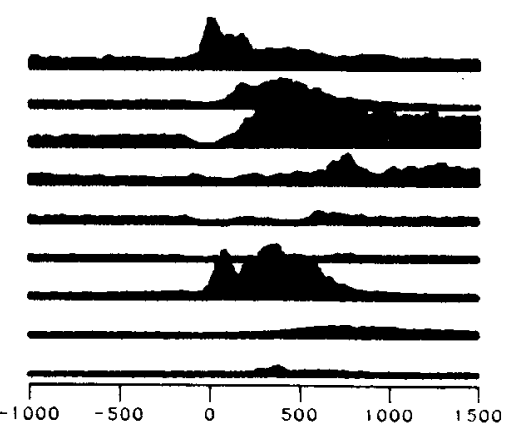

17

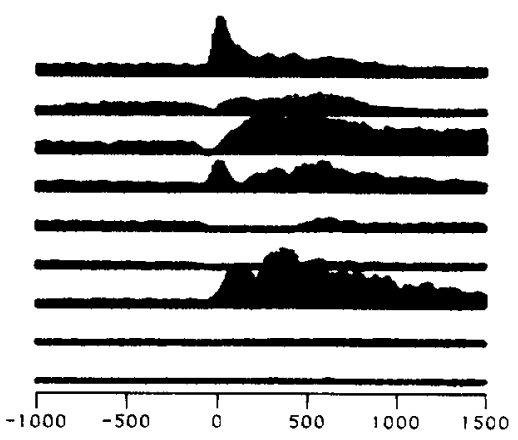

18

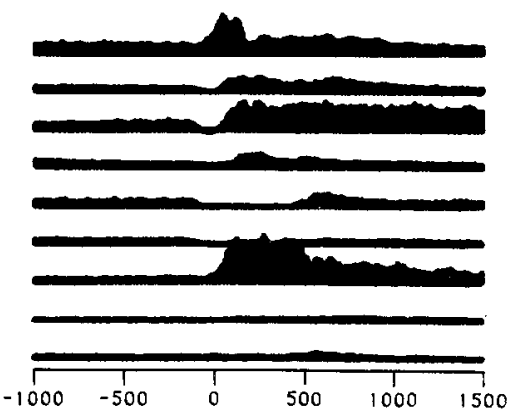

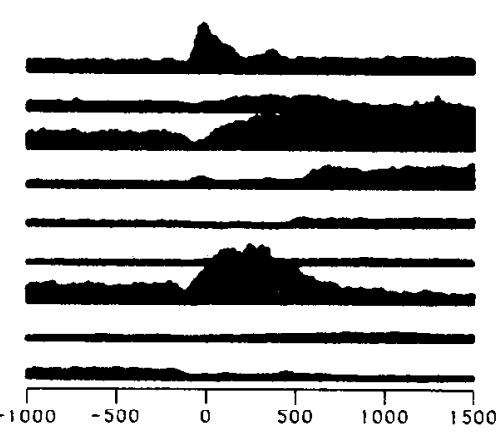

26

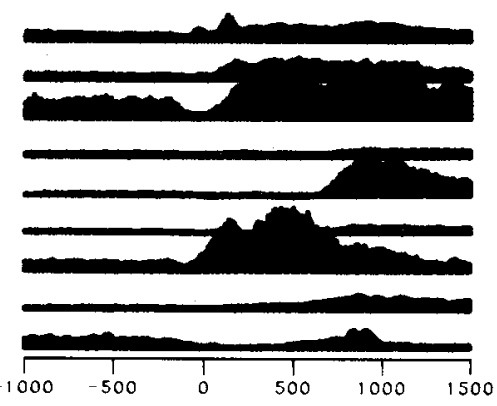

27
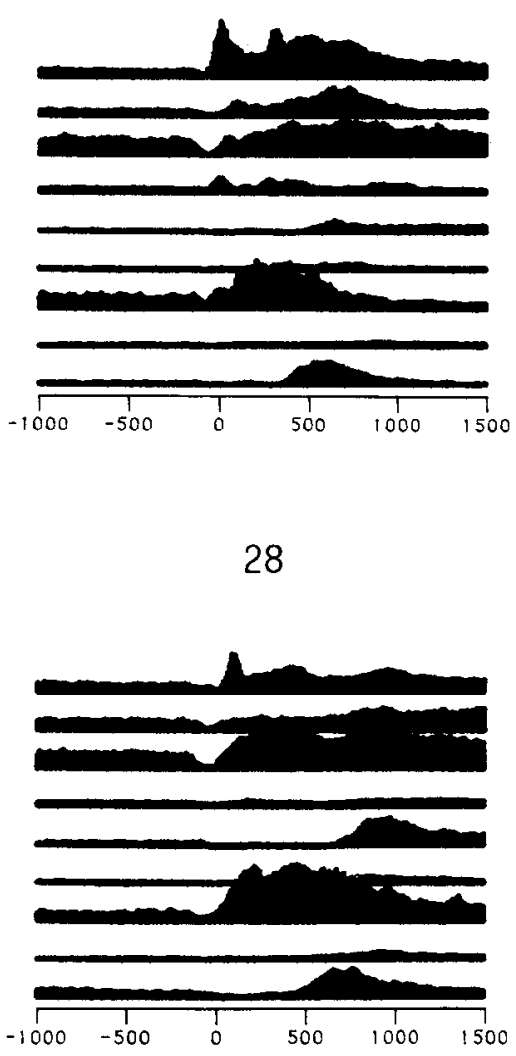
Table 1. Directional composition of the population of motor cortical cells studied in the task

\begin{tabular}{lc} 
Cells & Number (\%) \\
\hline Total number of cells & 176 \\
Cells studied in 3 parts of space & $136(77.3)$ \\
Directional & $134(98.5)$ \\
in 3 parts of space & $101(75.4)$ \\
in 2 parts of space & $25(18.7)$ \\
in 1 part of space & $8(6.0)$ \\
Nondirectional & $2(1.5)$ \\
Cells studied in 2 parts of space & $27(15.3)$ \\
Directional & $26(96.3)$ \\
in 2 parts of space & $21(80.8)$ \\
in 1 part of space & $5(19.2)$ \\
Nondirectional & $1(3.7)$ \\
Cells studied in 1 part of space & $13(7.4)$ \\
Directional & $9(69.2)$ \\
Nondirectional & $4(30.8)$
\end{tabular}

deltoid, clavicular deltoid, and pectoralis. For the remaining muscles (cranial trapezius, long head of triceps, biceps, and teres major), there were no significant interactions (ANOVA, $F$-test $p>0.05$ ) between movement direction and region of space where movements were made. Since movements in the task were accomplished by the synergistic action of all these muscles, it suffices that the activity of at least 1 muscle changes when movements of similar direction are made across the work space to conclude that the pattern of synergy changes accordingly. The data showed that in all 8 triplets of movement directions tested the patterns of synergy were modified by virtue of significant changes occurring in the activity of at least 2 muscles.

\section{Neurophysiological studies}

\section{Directional properties of motor cortical neurons}

A total of 176 arm-related cells were studied in the task in the course of 73 microelectrode penetrations in the motor cortex of 4 hemispheres in 3 monkeys. During neural recording, the animals performed the task using the arm contralateral to the recording site. All data were later referred to the right arm by appropriate transformations. The location of microelectrode penetrations in the precentral gyrus is shown in Figure 7. Penetrations were all in area 4 , as judged by the absence of a granular layer IV and by the presence of giant pyramidal cells in layer VI, and in the caudalmost part of area $6(6 \mathrm{a} \alpha$ of Vogt and Vogt, 1919; see Wiesendanger, 1981, for a review).

Since our main purpose was that of studying the directional properties of motor cortical cells across space, an ANOVA was first performed to select those cells which modulated their activity in a significant way with movement direction independently of where in space the movements were performed. Table 1 shows that 169 out of the $176(96.0 \%)$ cells studied were directional (ANOVA, $p<0.05$; TET epoch). Furthermore, the
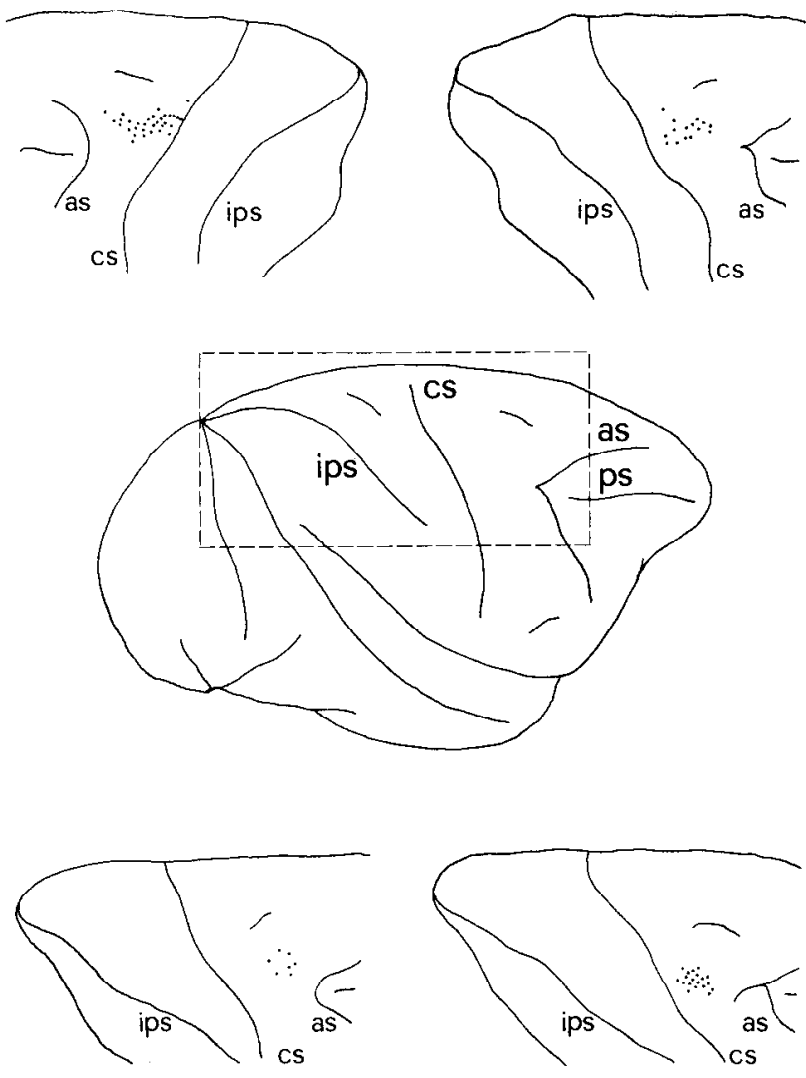

$2 \mathrm{~cm}$

Figure 7. Entry points of microelectrode penetrations $(n=73)$ shown in lateral views of 1 left and 3 right hemispheres of the macaque monkeys. Detail figures represent the region outlined in the brain figurine. $P_{S}$, as, cs, and ips indicate principal, arcuate, central, and intraparietal sulci, respectively.

degree to which these cells maintained their directionality throughout the entire work space was determined. Table 1 shows that of the 134 directional cells studied throughout the work space, $101(75.4 \%)$ were directional in all 3 parts of the space tested, $25(18.7 \%)$ of them were directional in only 2 (normally adjacent) parts of space, while $8(6 \%)$ of cells were directional in only 1 part of space. Cells directional in more than 1 part of space represent the usable data for our study. A multiple regression was then performed for each directional cell to determine whether the frequency of discharge varied in the way predicted by the tuning function described in Materials and Methods. This tuning function is that adopted by Schwartz et al. (1988). For the directional cells, the rate of discharge showed a good fit (multiple regression, $F$-test, $p<0.05$ ) to the model in the left, center, and right parts of the work space considered separately ( $93 \%$ of cell-cube combinations). For the remaining $7 \%$ of cases, the rate of discharge could not be explained by the tuning function adopted, and these cell-cube combinations were not further

Figure 6. EMG activity of 9 proximal muscles recorded during the task. Activity is shown for triplets (5-15-25, 6-16-26, 7-17-27, 8-18-28) of movements having similar directions but performed within different parts of space. In each panel, from top to bottom, data refer to the activity of caudal trapezius, cranial trapezius, spinal deltoid, clavicular deltoid, long head of triceps, lateral head of triceps, biceps longus, teres major, and pectoralis. Conventions and symbols as in Figure 5. 

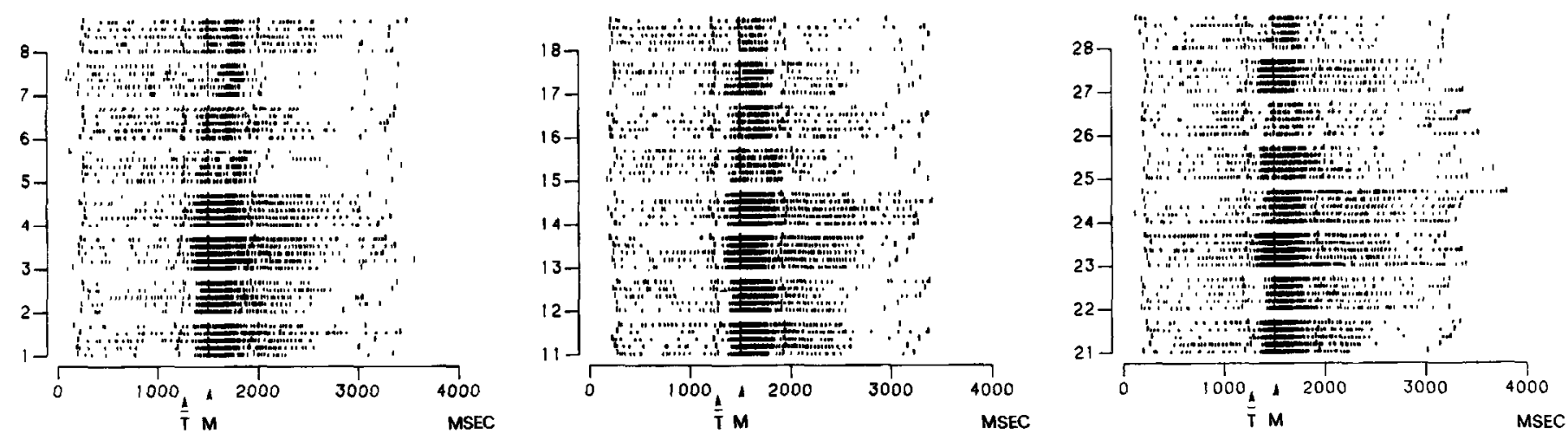

Figure 8. Impulse activity of a motor cortical cell (area 4) recorded during the task. Rasters of 5 replications for every movement direction within the 3 different parts of the work space were aligned to the movement onset $(M)$. Target onset is indicated by $T$. Longer vertical bars indicate, from left to right, beginning of the trial, target presentation, movement onset, beginning, and end of target holding time. Numbers on the vertical axes indicate directions of movement as labeled in Figure 1. This cell was directional within the entire work space.

analyzed. These results are in agreement with the observations by Schwartz et al. (1988).

Figure 8 shows the activity of an individual neuron of area 4 recorded while the monkey performed the task. This neuron was directional in all parts of the work space (ANOVA, $p<$ $0.0005)$, and its discharge rate fit the tuning function according to the above criteria. This figure illustrates how for many triplets of movement directions (1-11-21;2-12-22;3-13-23) the frequency and sign of activity, its time course, and its temporal relation to the movement onset did not change notably, while for the remaining triplets, discharge characteristics changed in some respects. For instance, when movements in directions 7-17-27 were made, both frequency of discharge and temporal relations of cell activity to movement onset varied across space. This is illustrated in the histograms of Figure 9. The motor cortical cell illustrated in Figure 10 was, instead, highly directional on the central (ANOVA, $p<0.0005$ ) and right (ANOVA, $p<0.0005$ ) parts of the work space but showed little modulation when movements were performed in the left part of the space; here, its activity did not change significantly with direction of movement (ANOVA, $p>0.05$ ). For this cell, as for the cell in Figure 8, movements of similar direction made in different parts of the work space resulted in changes in firing rate. A 2-factor ANOVA was performed on the discharge rate of all cells directional in 3 parts of the work space. This analysis was aimed at determining the number of cells showing significant changes in their discharge rate when movements were made within different parts of space. The results indicated that out of 136 cells studied, $118(86.8 \%)$ showed significant changes (interaction term, $p<0.05)$, while for the remaining 18 cells $(13.2 \%)$, the changes were not significant (interaction term, $p>0.05$ ).

The change in firing frequency observed for movements made in different parts of the work space was reflected in the 3-D orientation of the preferred direction of the cells as illustrated in Figure 11 for a single motor cortical cell. The preferred directions computed from cell activity in the left, center, and right parts of the work space are shown as vectors with origins in 3 Cartesian coordinate systems centered at the origins of movement. It can be seen that these values differ across space, determining a rotation of the cell's preferred direction mainly in the horizontal plane (around the $Z$ axis). The amount of rotation, and occasionally its sign, was different for different cells. Since no conclusions could be drawn from the analysis of single cells, an analysis of the entire population of motor cortical cell preferred directions was undertaken.

\section{Analysis of the spatial orientation of motor cortical cell preferred directions across space}

$\Lambda \mathrm{s}$ a first step in the population analysis, the preferred directions of all cells studied in the task were computed from cell activity
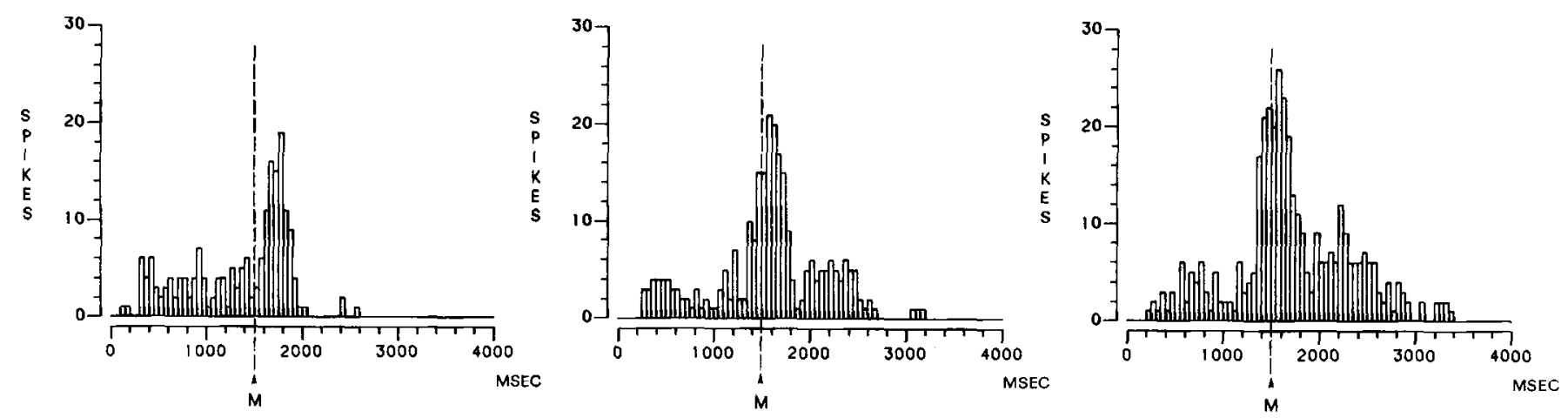

Figure 9. Perievent time histograms showing cell activity relative to the movement onset $(M)$ in directions 7,17 , and 27 for the ccll shown in Figure 8. Histograms are generated from 5 replications of cell activity in each movement direction: 

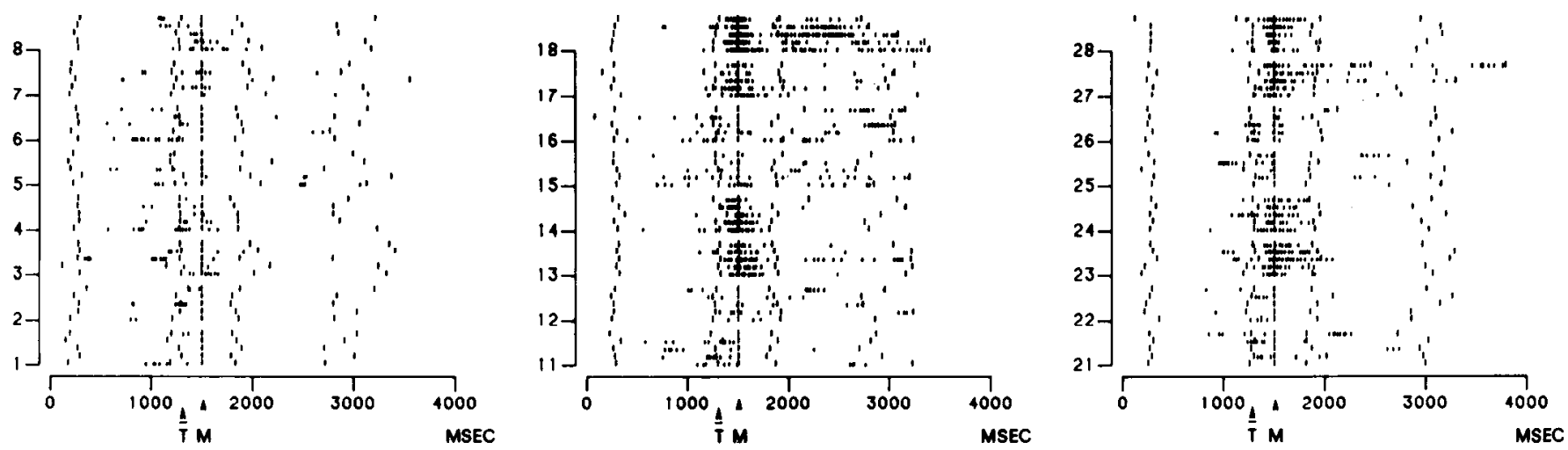

Figure 10. Impulse activity of a motor cortical cell (arca 4) recorded during the task. This cell was directional in the center and right parts of the work space only. Abbreviations and symbols as in Figure 8.

during the TET and expressed as vectors of given 3-D orientation. Their distribution is shown in Figure 12, in a 3-D plot and in Figure 13 in the form of 2-D equal area projection plots (Watson, 1983, p. 22). These latter were obtained first by representing, for each of the 3 parts of the work space, the cells' preferred directions as vectors of unit length originating from the movement origin and thus defining a unit sphere. This sphere was then "flattened" onto a plane and the positions of the vector end points projected onto the plane. The projection was made in such a way that areas on the sphere are represented by equal areas on the plane and was performed separately for upper and lower hemispheres and for each part (left, center, right) of the work space tested. The upper and lower hemispheres represent the upper and lower parts of the work space, relative to the movement origins. There were 3 salient features of these distributions. First, the preferred directions of the cell population covered the entire 3-D continuum, in agreement with the observations of Schwartz et al. (1988). Second, this was true for all 3 parts of the work space where cells were studied. Third, the spatial orientations of the preferred directions shown in the left, center, and right parts of space were correlated (Fisher and Lee, 1983; spherical correlation coefficient, $r=0.312, p<0.002$ for left-right correlation; $r=0.505, p<0.002$ for left-center correlation; $r=0.485, p<0.002$ for center-right correlation). These data indicate that the correlations between the orientations of preferred directions in adjacent parts of space are higher than that between nonadjacent parts of space. Despite the relatively low value of the coefficients, in all cases the correlations between the spatial orientations of preferred directions were highly significant.

These data represent, at the population level, a base to uncover possible changes of cells' preferred directions as a con- sequence of the behavioral conditions imposed by the task. In this search, we performed a "local" spatial analysis by first selecting 8 sectors in the center part of the work space, 4 lying in its upper portion (upper hemisphere; Fig. 14) and 4 in its lower portion (lower hemisphere; Fig. 15). The cells having preferred directions lying within these sectors were identified and their preferred directions were followed when the animals were working in the left and right parts of the work space. The equal area projection plots show that the distributions of cells' preferred directions changed across the space. The distributions of cells' preferred directions in left and right parts of the space showed, in fact, a significant scatter when compared with their distribution in the central part of space. The next step was to quantify this change, and most importantly, to determine whether it occurred in an orderly fashion. First, for each cell, the angular differences between pairs of preferred directions lying in adjacent (left-center, center-right) and nonadjacent (left-right) parts of space were computed. The distributions of these angular differences were expressed as frequency histograms (Fig. 16). As shown, the median angular difference between the preferred directions of the same cells tested in the left and center part of the work space was $28^{\circ}$, and similar results were obtained when pairs of preferred directions were compared between center and right parts of space (median angular difference, $23^{\circ}$ ). The median angular difference was $43^{\circ}$ when the difference was computed between the orientation of preferred directions in the nonadjacent right and left parts of the work space. The similarity of the angular differences between orientations of preferred directions in adjacent parts of space and the larger angular difference observed between preferred directions from nonadjacent parts of space suggests the existence of an orderly shift of the orientation of cells' preferred directions. These data do not reveal,
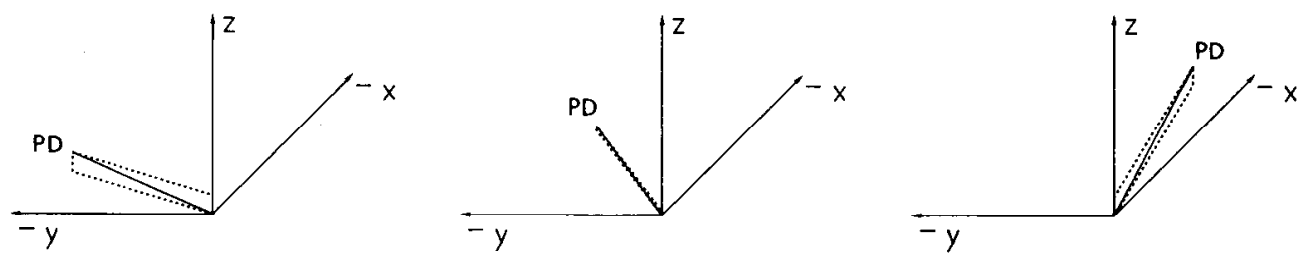

Figure 11. Spatial orientation of the vectors representing the preferred directions $(P D)$ of an individual motor cortical cell in the left $(L)$, center $(C)$, and right $(R)$ parts of the work space represented within 3 Cartesian coordinate systems centered at the origins of the movements performed in the task. 

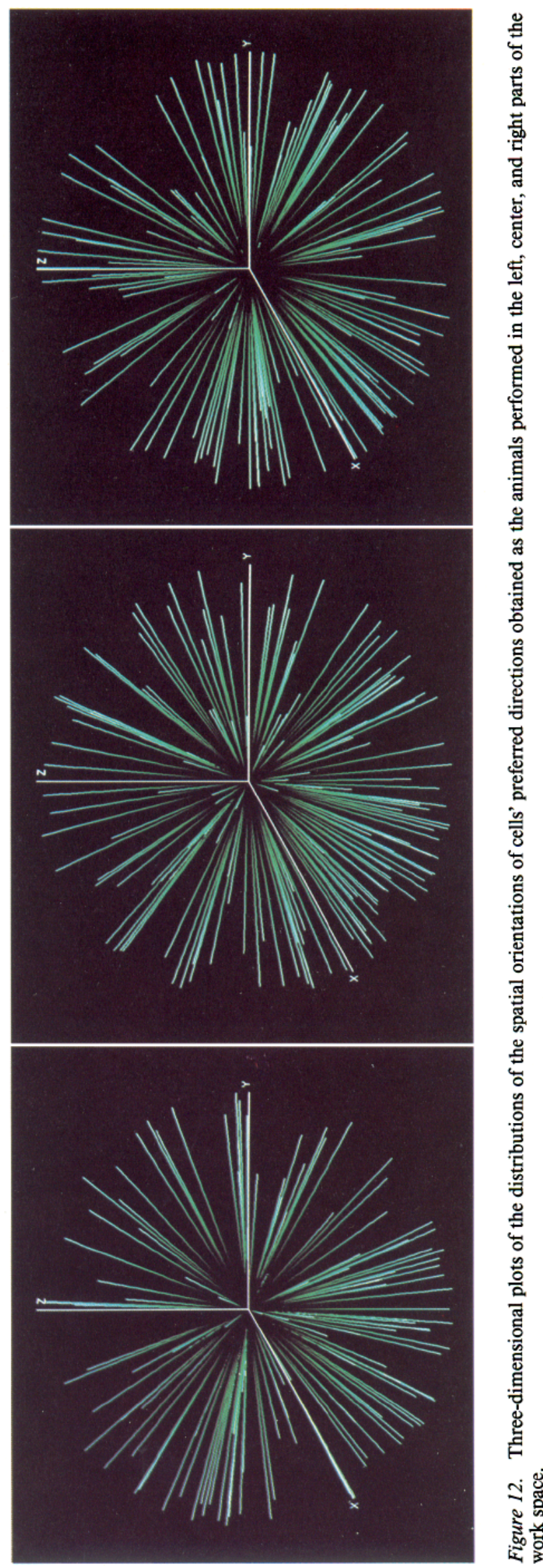
UPPER HEMISPHERES ( TET )
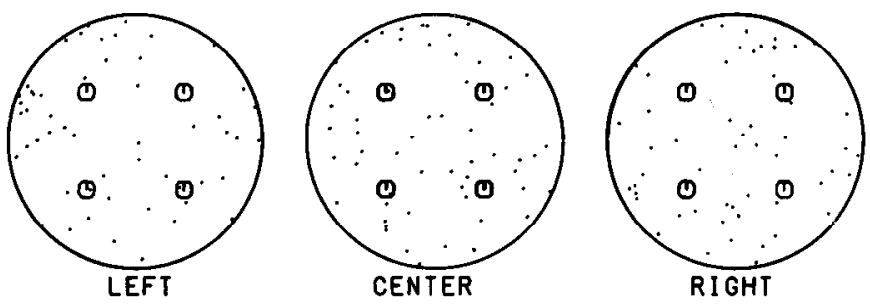

LOWER HEMISPHERES ( TET )
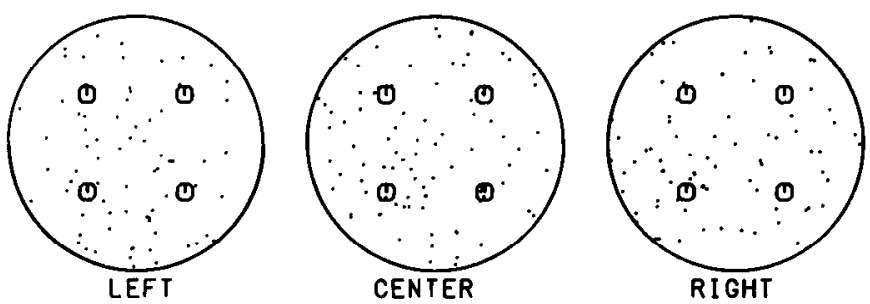

Figure 13. Distribution of cell preferred directions (dots) in the left, center, and right parts of the work space in the form of equal area projection plots. Projections of preferred directions on upper and lower hemispheres (parts of the work space) are shown separately. The points in the upper and lower hemispheres represent the preferred directions pointing up and down, respectively, relative to the origin of movement. On each plot, points at 6 and 12 o'clock represent preferred directions pointing toward and away from the animal's body, respectively. Points near 3 and 9 o'clock are preferred directions pointing to the right and left, respectively, relative to the animal's midline. Open circles, target positions; $T E T$, total experimental time. See text. however, along which spatial axis the change in orientation occurred. To address this question, a spherical regression analysis (Jupp and Mardia, 1980) was introduced. Given 3 sets of vectors (the preferred directions of the cell population studied in the left, center, and right parts of the work space), this analysis determines, around each of the axes of a Cartesian coordinate system, the amount of rotation necessary to bring 1 set into maximal spatial coincidence with another. The results of the spherical regression, shown in Table 2, indicate that the main rotation occurred around the $Z$ (vertical) axis. To bring into coincidence the different sets of preferred directions, a rotation around the vertical axis of $12.6^{\circ}(95 \%$ confidence interval (CI) $\left.=\left[8.9^{\circ}, 16.2^{\circ}\right]\right)$ was necessary when center and left parts of space were considered, a similar amount of rotation $\left(11.8^{\circ} ; \mathrm{CI}=\left[7.0^{\circ}\right.\right.$, $\left.16.4^{\circ}\right]$ ) was needed for the center-right coincidence, while a larger rotation $\left(37.1^{\circ} ; \mathrm{CI}=\left[29.3^{\circ}, 43.1^{\circ}\right]\right)$ brought into coincidence preferred directions from the left-right nonadjacent parts of the work space. The data presented in Table 2 are the result of rotations first around the $X$ and then around $Z$ and $Y$ axes. Due to the fact that the rotations around the $X$ and $Y$ axes were relatively small, changing the order of rotation did not have a significant effect upon the resulting angles. This rotation of spatial orientation of cell preferred directions around the $Z$ axis is also represented in Figure 17, as a 3-D plot. It can be seen that while the trajectory followed in space by the preferred direction vectors may be different in magnitude, oricntation, and somctimes even in sign for different cells, at the population level the overall result is a rotation occurring mainly around the vertical axis. The results of the spherical regression analysis were substantiated by computing for each individual cell, the differences

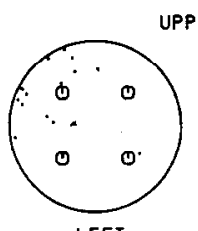

UPPER HEMISPHERES ( TET)

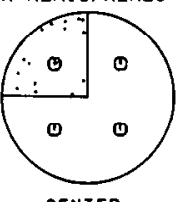

CENTER

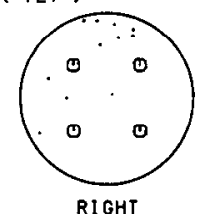

LOWER HEMISPHERES ( TET )
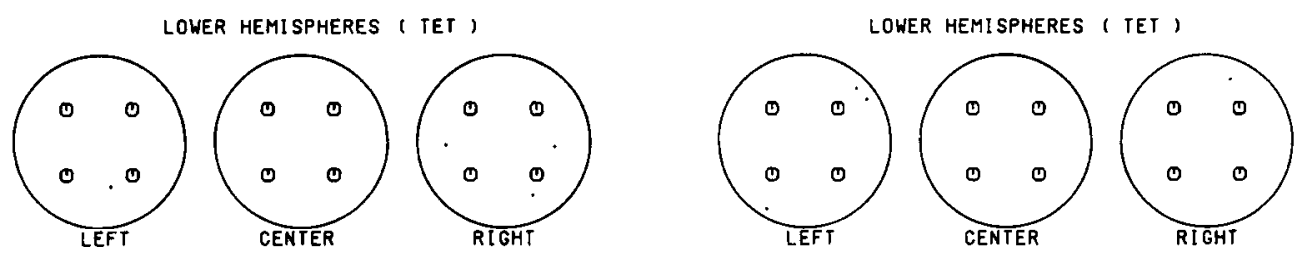

LOWER HEMISPHERES ( TET ,
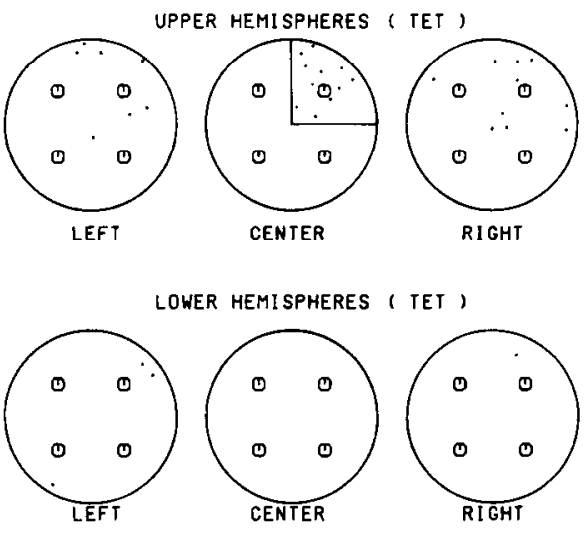
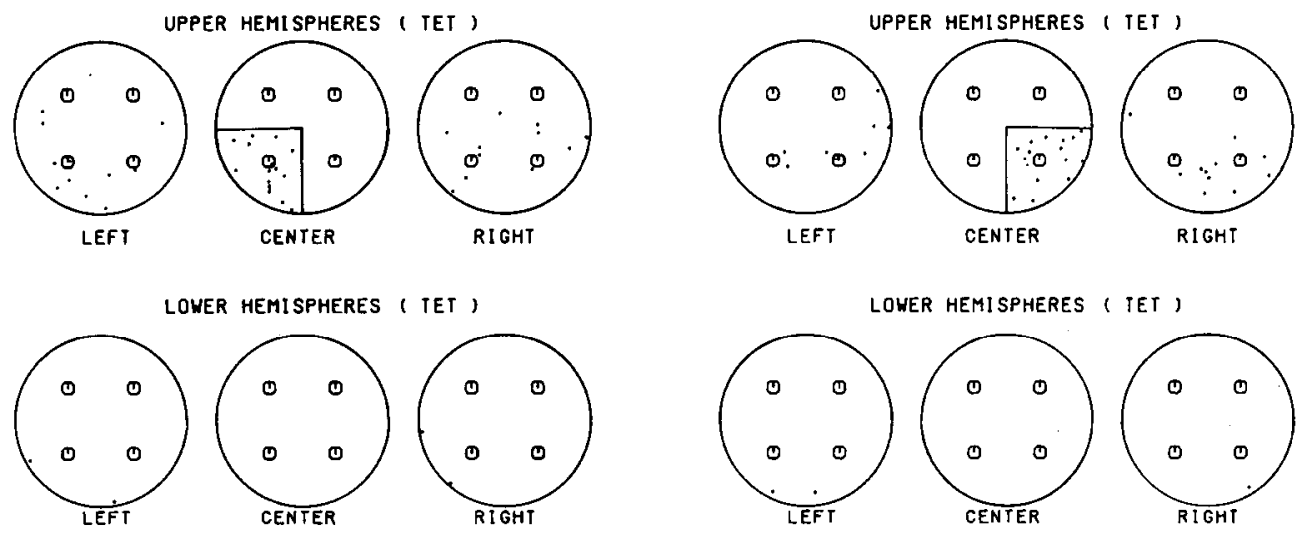

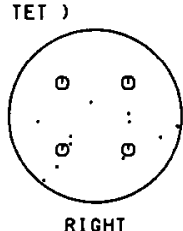

LOWER HEMISPHERES ( TET )

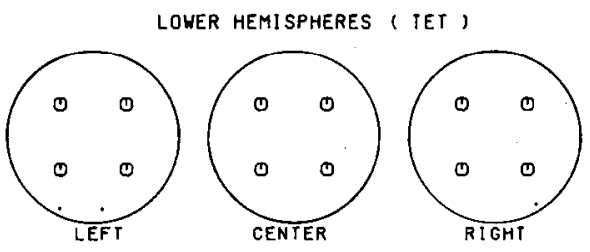

Figure 14. Upper hemispheres, cells with preferred directions lying in 4 sectors of the upper-center part of the work space were selected from the equal-area projection plots and the spatial orientation of their preferred directions was "tracked" as the animals performed within the left and right parts of the work space. See text for further explanation. Conventions and symbols as in Figure 13. 

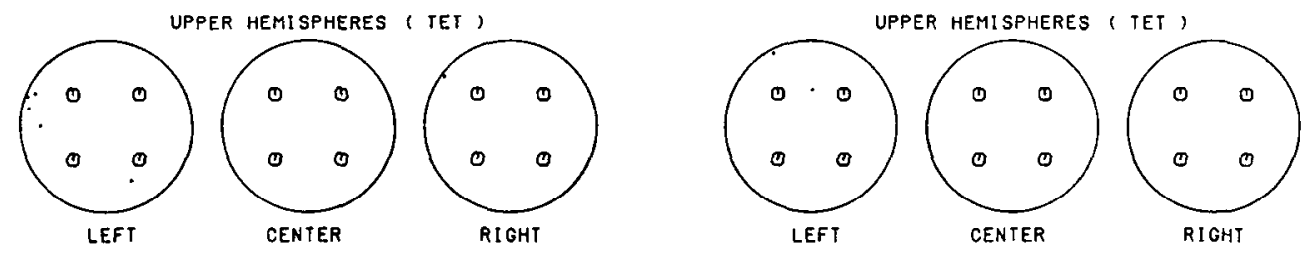

LOWER HEMISPHERES (TET)

LOWER HEMISPHERES ( TET)
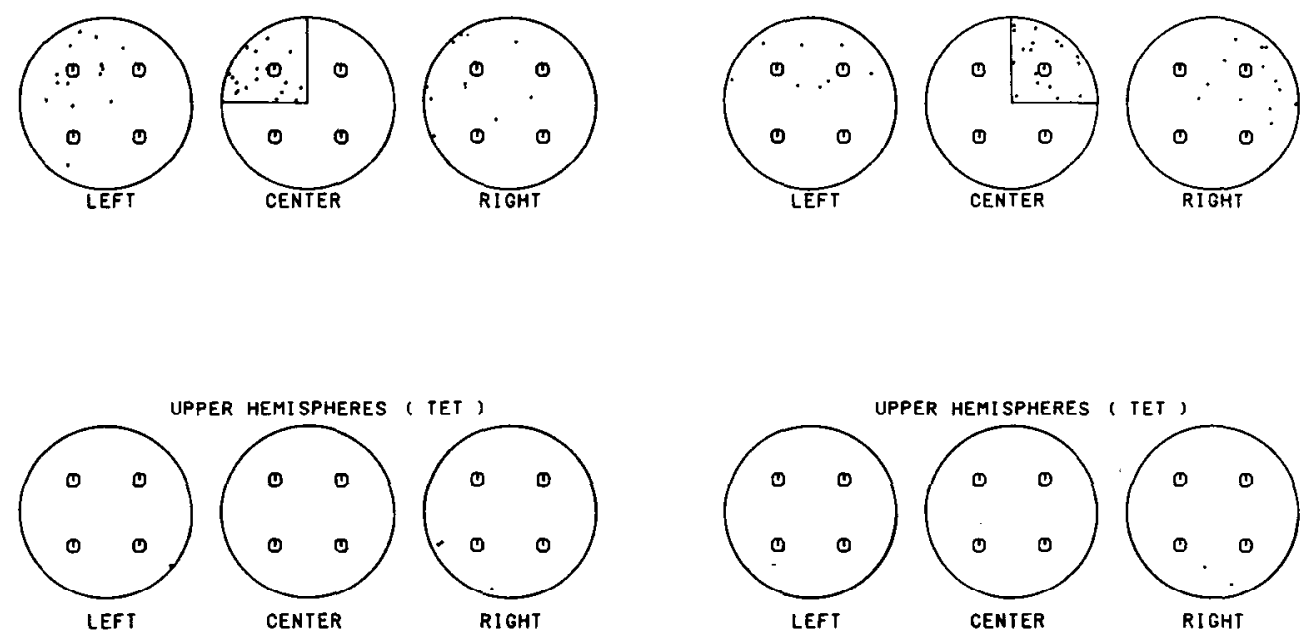

LOWER HEMISPMERES ( TET )

LOWER HEMISPMERES ( TET)

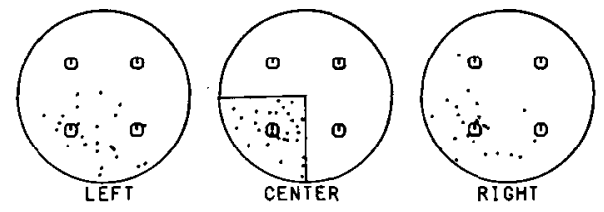

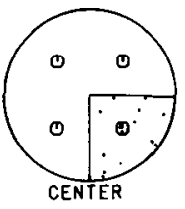

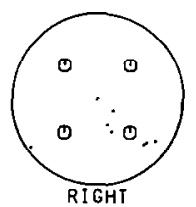

Figure 15. Lower hemispheres, cells with preferred directions lying in 4 sectors of the lower-center part of the work space were selected from the equal-area projection plots and the spatial orientation of their preferred directions was "tracked" as the animals performed within the left and right parts of the work space. See text for further explanation. Conventions and symbols as in Figure 13.
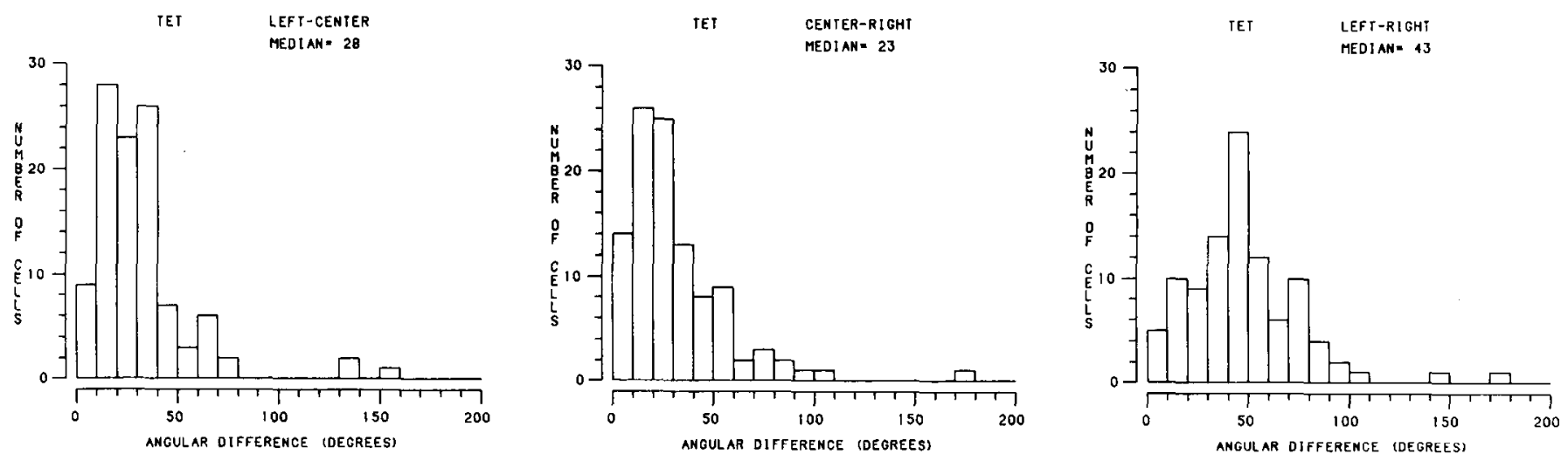

Figure 16. Frequency distributions of the angular differences between the spatial orientation of cell preferred directions across the work space. These differences were computed between preferred directions lying in the left and center, center and right, left and right parts of the work space. $T E T$, total experimental time.

Figure 17. Three-dimensional plot showing the rotation of the spatial orientation of the cells' preferred directions as movements of similar directions were made in different parts of space. Each red line represents the trajectory followed by the preferred direction vector of an individual motor cortical neuron, with the tail (darkest part) indicating the original position of the vector in the left part of the animal's work space and the head (lightest part) its position in the right part of space. The rotation is vicwcd from 6 different angles: $0^{\circ}$ (top left), $60^{\circ}$ (top right), $120^{\circ}$ (center left), $180^{\circ}$ (center right), $240^{\circ}$ (bottom left), $300^{\circ}$ (bottom right). In each plot brighter vectors indicate preferred directions lying in the portion of the sphere nearest to the observer. In the $0^{\circ}$ view, the observer's perspective coincides with that of the monkey performing the task. 

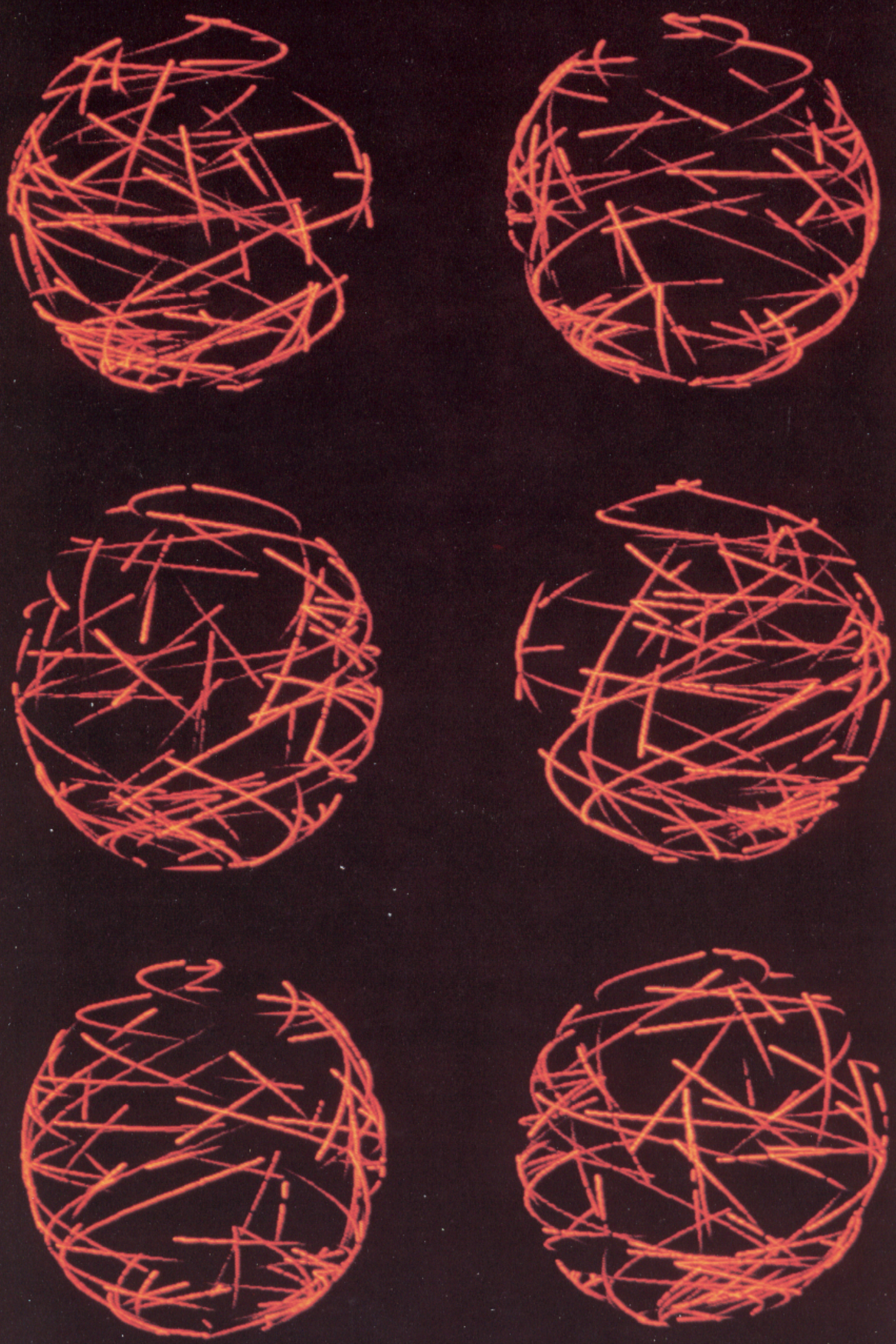

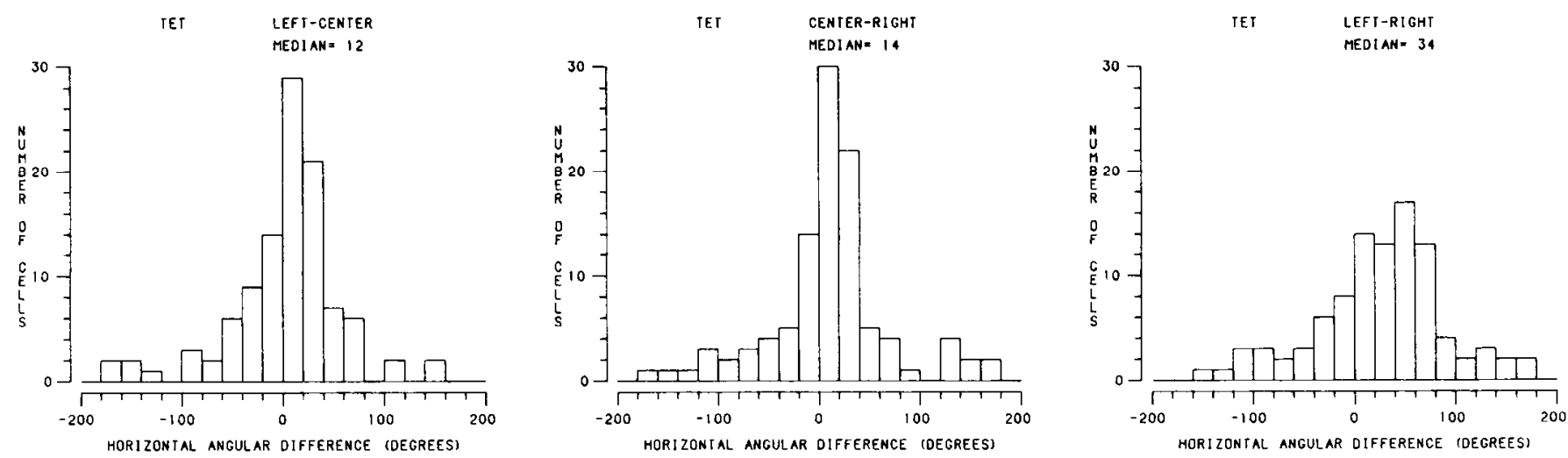

Figure 18. Frequency distributions of the horizontal angular differences between the spatial orientations of cells' preferred directions across the work space. These differences were computed between preferred directions lying in the left and center, center and right, left and right parts of the work space. TET, total experimental time.

between its left-center, center-right, and left-right preferred directions in the horizontal planes. Histograms of these data (Fig. 18) show that, while there is substantial spread of the distribution around the median values, the data are consistent with the spherical regression: median angular differences in the horizontal plane (and therefore around the vertical axis) are $12^{\circ}$ between left and center, $14^{\circ}$ between center and right, and $34^{\circ}$ between left and right.

Due to the horizontal arrangement of the 3 cubcs of the behavioral apparatus, movements from 1 part of the work space to another involved mainly horizontal rotations of the shoulder joint. Horizontal rotations of the shoulder joint of approximately $20^{\circ}$ from left to center and $18^{\circ}$ from center to right were necessary to move the hand from the center of 1 cube to the center of the other. These angles varied slightly in the 3 animals used due to variations in shoulder width and arm length. Thus, the horizontal rotation observed in the population of preferred direction vectors of motor cortical cells follows closely the rotation necessary at the shoulder joint to bring the arm into the different parts of the work space.

\section{Neuronal population vector across space}

The existence of a broad relationship between direction of movement and cell activity in the motor cortex, also observed in this study, has led to the proposition that direction of movement is represented by a population rather than a single-cell code (Georgopoulos et al., 1983, 1986, 1988). In these studies, population vectors computed from real data proved to be good predictors of arm movement direction. In our study we asked the question whether the population vector was a good predictor of movement direction regardless of where in space the animal

Table 2. Spherical regression analysis

\begin{tabular}{llll} 
& $\Phi$ & $\Theta$ & $\Psi$ \\
\hline Left-center & $12.6^{\circ}$ & $5.4^{\circ}$ & $3.6^{\circ}$ \\
Center-right & $11.8^{\circ}$ & $1.2^{\circ}$ & $3.8^{\circ}$ \\
Left-right & $37.1^{\circ}$ & $4.9^{\circ}$ & $7.7^{\circ}$
\end{tabular}

$\phi$, rotation around $z$ axis; $\theta$, rotation around $y$ axis; $\psi$, rotation around $x$ axis. was performing. To address this question, we computed the population vector for all movement directions in each of the 3 regions of the work space. The average angular difference between population and movement direction vectors was found to be $26.9^{\circ}, 25.1^{\circ}$, and $12.0^{\circ}$ in the left, center, and right parts of the work space, respectively. These data are similar to those reported in previous studies (Georgopoulos et al., 1988; Kettner et al., 1988) and, in addition, indicate that the population vector remains a valid descriptor of movement direction across space. The smaller angular difference between the population and movement vectors observed in the right versus center and left regions of the work space may depend, in part, upon the relatively small sample size used in this analysis.

\section{Discussion}

The objective of this study was to describe the motor cortical mechanisms underlying the planning and execution of arm movements in different directions. Toward this end, the activity of single motor cortical neurons was studied and compared while monkeys made arm movements of parallel directions within different parts of extrapersonal space. The behavioral paradigm was aimed at maintaining a similar direction of movement trajectories across space while varying the patterns of muscular activity and joint angles necessary for these movements. The achievement of such a dissociation between intrinsic and extrinsic frames of references was substantiated by the consistent changes in the activity of most of the muscles participating in the generation of the movements tested as the animal worked in different regions of the space. Since the movements in this task were accomplished through the synergistic action of many muscles, attention was devoted to the analysis of the patterns of synergy. These patterns changed as a result of changes in the activity of many of the component muscles. The degree of change in muscle pattern and the muscles responsible for these changes varied as a function of the direction of movement.

There are several main points to be discussed concerning the motor cortical cell activity recorded during the task employed in this study. The first concerns the relationship of motor cortical cell activity to direction of movement, the model used to express this relation across space, and the comparison with other studies. The second refers to the analysis of the way in which the directional properties of motor cortical cells changed according 
to the behavioral conditions of the task. The third point concerns the relationship of the observed motor cortical activity to possible muscle state variables. We will then consider the relevance of the changes in cortical activity across the work space for a description of a coordinate system representing direction of arm movement in the motor cortex. Finally, we will discuss the relevance of population codes for describing the motor cortical representation of arm movement in space.

\section{Relations of motor cortical cell activity with direction of movement in the 3-D space}

Our data base consisted of a population of motor cortical cells whose activity was related to movement of the upper arm at the shoulder joint. Neurons showing significant variations in activity with movement direction (and, therefore, considered as directional) represented the large majority (96.0\%) of our samplc. This is in agreement with previous studies of arm movement in 2-D (Georgopoulos et al., 1982; Kalaska et al., 1989) and 3-D (Schwartz et al., 1988) tasks. Our data are also consistent (although not directly comparable) with those studies using unidimensional tasks which have stressed the importance of movement direction as a determinant of motor cortical cell activity (Schmidt et al., 1975; Thach, 1978; Crutcher and Alexander, 1987; see Evarts, 1981, for a review). The existence of directional modulation in our study was, for most cells, observed throughout the work space, suggesting that a single population of directional motor cortical cells can account for movements in different directions within different parts of the extrapersonal space. However, a small number of motor cortical neurons underwent a loss of directional specificity for movements in given "nonpreferred" parts of space. The mechanism underlying this phenomenon remains to be elucidated.

\section{Comparison of directional properties of motor cortical cells for movements of similar direction within different parts of work space}

The main result of this study was that, when movements of similar direction were made within different parts of space, the spatial orientation of the preferred directions of motor cortical neurons changed significantly. This modification had a spatial order, following closely the rotation of the shoulder joint in space. The fact that this rotation occurred mainly around the vertical axis was most probably a consequence of the behavioral condition imposed by the task which required the animals to make movements within different parts of space separated in the horizontal plane. Thus, a rotation around the vertical axis (horizontal plane), with little joint displacement around the other axes of rotation, was necessary to bring the arm to the appropriate part of space. The results of the spherical regression analysis were unequivocal in showing that the rotation required to bring the cells' preferred directions from different left-right regions of the work space into coincidence was many times larger in the horizontal than in the other 2 planes of rotation. From this one can predict that, if the animal would have been required to make arm movements in parts of space requiring rotations around a different axis, cell preferred directions would have shown more rotations in other planes. This hypothesis can be experimentally tested. It is interesting that this shift of spatial orientation of cell preferred directions was evident even in the early phase of the reaction time, suggesting that it was the result of a central command. Finally, it is worth noting that Humphrey et al. (1970), Thach (1978), and Murphy et al. (1982) have suggested the existence of relations of motor cortical cell activity with the angular displacements of the joints involved in arm movements.

\section{Relationship of motor cortical activity to patterns of muscle synergies}

Corticospinal neurons project with divergent axons to different motoneuronal pools (Shinoda et al., 1979, 1981) and engage them with different strengths (Fetz and Finocchio, 1975; Fetz and Cheney, 1978, 1980; Cheney and Fetz, 1985; Lemon et al., 1986). It is reasonable to assume that the relationships between motor cortical cells and spinal motoneurons are constant combinations by which appropriate synergies of muscle activity are selected to generate a movement in a given direction. These muscle synergies can be represented in vector form: the vector's orientation corresponds to the sum of the vectors parallel to the axes of the individual muscles of the synergy. The orientation of such a synergy vector will not remain constant with respect to an extrapersonal coordinate system but will rotate with the position of the arm in space. Our data are consistent with the hypothesis that motor cortical cells command muscle synergies. In this respect, it should be noted that Mussa-Ivaldi (1988) has proposed a theoretical model by which motor cortical cells can encode muscle state variables.

\section{Cortical representation of a coordinate system for planning and execution of arm movement in extrapersonal space}

To consider motor cortical activity as merely reflecting muscle synergy information would overlook an important point. In the processes which we have studied, the flow of information is from cortex to muscle and, therefore, the cortex must be able to transform the visual information relating to target location and the proprioceptive input concerning the arm position in space into a representation which is appropriate for commanding muscle activity. In our experimental set-up, the preferred direction of each cell was studied in the 3 parts of space where the animals performed. The experimental data show how the orientations of cells' preferred directions rotated when movements of similar direction were made within different parts of space. This suggests the existence of a mechanism which can combine more than one type of information. The first type of information contains the representation of movement direction, is acquired by the animal using visual information, and is probably represented within an extracorporeal frame of reference. The second type of information is represented by the position of the arm in space; it is acquired through proprioceptive pathways and probably reflects an active motor set. Exactly where within the distributed motor system the actual combination of information occurs is not indicated by our data; however, we have clearly observed the result of this combination at the level of the primary motor cortex.

In our experimental paradigm and in the model adopted to describe cell activity, movement direction is represented in a vectorial form within a Cartesian coordinate system centered at the origin of movement. The choice of this model rests on the experimental conditions: the targets being positioned in extrapersonal space, they can be located only by using visual information. The vectors representing movement direction in the work space have constant relations to head and torso coordinates, given that the animal's head was fixed and that all movements were performed without significant movement of the torso. The position in space of the arm can be represented by a 
sccond set of vectors. The relationship of this set of vectors to that representing movement direction is variable, given that 1 rotates relative to the other when the origin of movement changes. The rotation of these 2 sets of vectors, 1 relative to the other, can be computed by simply specifying the position of the arm in space. At neural levels, motor cortical neurons (Georgopoulos et al., 1984b; Kettner et al., 1988; Kalaska et al., 1989; this study) have access to this type of information.

We hypothesize that the combination of these 2 signals determines the rotation in space of the orientation of cell preferred directions. Assuming that the muscle synergy vector is a static property of corticospinal neurons, an optimal motor command for moving the arm in a desired direction would require the "projection" of the movement direction vector onto a coordinate system centered on the shoulder joint and representing the natural coordinates of the arm. The mechanism observed at the motor cortical lcvel, by combining information relative to movement trajectory and arm orientation in space, can compute this "projection" which can be obtained by a formal relation equivalent to the rotation of these sets of vectors, 1 relative to the other. This interaction of inputs can also be described through a formal model (Y. Burnod, P. B. Johnson, and R. Caminiti, unpublished observations). This model predicts that, as the position of the arm changes for making movements of similar direction across space, the cells' preferred directions should change accordingly. This prediction has been fulfilled by the experimental observations.

The data suggest that the visuomotor transformation studied in this task can be achieved by a mechanism which consists of a dynamic real-time combination of inputs which, by using information about the arm position in space, relates signals about movement trajectory to the motor output. This combination would provide a motor cortical output not yet coded in exclusive muscle coordinates; this last transformation can be performed within the highly specialized circuitry of the propriospinal system (Lundberg, 1979; Alstermark et al., 1981). Our data are in agreement with those of Georgopoulos et al. (1982) and Schwartz et al. (1988) in stressing the importance of movement trajectory as a determinant of motor cortical cell activity and also with recent studies (Kalaska et al., 1989) centered on the analysis of the interaction between dynamics and kinematics at motor cortical levels. In addition, our results specify the way in which information relative to the representation of movement trajectory can be efficiently combined at the cortical level, with that concerning the position of the arm in space. Interestingly, this mechanism resembles that described for visuomotor integration in the posterior parietal cortex of the macaque (Andersen et al., 1985; see Andersen and Zipser, 1988, for a review), whcrc ncurons in arca $7 \mathrm{a}$, by combining information relative to the retinal location of the visual stimulus and the position of the eye in the orbit, seem able to provide a code in craniotopic space.

The cortical operations described in this paper can be of interest also in relation to the problem of the central representation of visuomotor transformations. It is often assumed, in fact, that, when a reaching movement at visual targets located in the extrapersonal space is made, the initial planning occurs in the external coordinates of the physical world. A coordinate transformation of this Cartesian frame to a joint system of reference (inverse kinematics) is then necessary to produce the muscle torques (inverse dynamic) necessary to bring the hand to the desired position of space. The complexity of this computation has led to the proposition that there exist patterns of invariances and that these patterns may reveal the principles underlying motor control. Within this conceptual frame, the observation (Morasso, 1981; Abend et al., 1982; Flash and Hogan, 1985; Hollerbach and Atkeson, 1987; see Hogan, 1988, for a review) of the tendency to generate roughly straight trajectories when moving the hand between pairs of points in space has suggested that movement is coded in terms of hand trajectory in space. Coupling of shoulder and elbow motions during arm movements have been interpreted as indicating a coding mechanism in intrinsic frames (Soechting and Lacquaniti, 1981; Lacquaniti and Soechting, 1982; see Soechting and Terzuolo, 1988 for a review). Our experimental results indicate how a cortical area important for movement trajectory formation, like the motor cortex, can contain representations combining information from different domains. There is, in fact, at the motor cortical level an invariant relationship between cell preferred direction and arm position in space. This invariance suggests the existence of a mechanism by which any time the arm moves to bring the hand to a desired region of the work space, the frame of reference is reset to the new coordinates of the shoulder joint. Therefore, the motor cortex seems to develop an internal representation of space where the coding of hand trajectory would occur within a coordinate system centered on the shoulder joint. This interpretation is consistent with the results of recent behavioral studies by Soechting and Flanders (1989a, 1989b). These authors have studied the errors accompanying the execution of pointing movements at visual targets on the assumption that movement errors can be used to reveal the steps involved in the process of sensorimotor transformation. From these studies, Soechting and Flanders concluded that pointing at visual targets in the extrapersonal space occurs within a shoulder-centered coordinate system; the intervening errors can be attributed to the transformations required to represent target location and spatial orientation of the arm within a common frame of reference.

\section{Neuronal population approaches to the study of motor cortical function}

In this study an invariance was observed between motor cortical cell preferred direction and arm orientation in space. This invariance was achieved through changes in the spatial orientations of cell preferred directions as the orientation of the arm in space changed. These modifications in cell preferred directions differed in both magnitude and orientation for individual neurons. Despite this, the average effect of these individual changes, as assessed by the spherical regression, was a global rotation of cell preferred directions which paralleled the rotation of the arm. Thus, it should be stressed that the observed invariance was achieved at the population, rather than at the single-cell, level.

In previous studies, the broad relations of motor cortical cell activity to direction of movement have suggested that distributed processes are responsible for the coding of direction of movement (Georgopoulos et al., 1983, 1986, 1988). On the assumption that individual neurons make a vectorial contribution which is greater for movements with direction similar to their preferred direction, the vectorial sum of these contributions, the "movement population vector," was computed. This population vector proved to be a good predictor of the upcoming movement direction (Georgopoulos et al., 1983, 1986, 1988). In these studies, movement population vectors were computed from movements performed only within a single lim- 
ited region of the work space around the animal's midline. In our study, movement population vectors were computed from cell activity recorded in the left, center, and right parts of the work space. In all instances, they provided a very good description of the direction of movement regardless of where in space movement occurred. Population codes based on the same model have been used to describe direction of reaching also in cortical area 5 (Kalaska et al., 1983) and the cerebellum (Fortier et al., 1989). It is worth mentioning that the same model has been successfully adopted in interpreting the mechanisms underlying the coding of the direction of stimulus motion by parietal visual neurons (Steinmetz et al., 1987). Together, these data suggest that distributed codes underlie different functions controlled by the nervous system and support the general validity of the population vector hypothesis for understanding the treatment of directional information in the cerebral cortex.

\section{References}

Abend, W, Bizzi E, Morasso P (1982) Human arm trajectory formation. Brain 105:331-348.

Alstermark B, Lundberg A, Norrsell U, Sybirska E (1981) Integration in descending motor pathways controlling the forelimb in the cat. 9. Differential behavioral defects after spinal cord lesions interrupting defined pathways from higher centres to motoneurones. Exp Brain Res 42:299-318.

Andersen RA, Zipser D (1988) The role of the posterior parietal cortex in coordinate transformations for visual-motor integration. Can $\mathrm{J}$ Physiol Pharmacol 66:445-462.

Andersen RA, Essick GK, Siegel RM (1985) Encoding of spatial location by posterior parietal neurons. Science 230:456-458.

Cheney PD, Fetz EE (1980) Functional classes of primate corticomotoneural cells and their relation to active force. J Neurophysiol 44:773791.

Cheney PD, Fetz EE (1985) Comparable patterns of muscle facilitation evoked by individual corticomotoneuronal (CM) cells by single intracortical microstimuli in primates: evidence for functional groups of CM cells. J Neurophysiol 53:786-804.

Cochran WG, Cox GM (1957) Experimental designs, 2nd ed. New York: Wiley.

Crutcher MD, Alexander GE (1987) Comparison of movement-related neuronal activity in primate motor cortex and putamen. Soc Neurosci Abstr 13:224.

Draper NR, Smith H (1981) Applied regression analysis, 2nd ed. New York: Wiley.

Efron B (1979) Bootstrap methods: another look at the jackknife. Ann Stat $7: 1-26$.

Evarts EV (1968) Relation of pyramidal tract activity to force exerted during voluntary movements. J Neurophysiol 31:14-27.

Evarts EV (1969) Activity of pyramidal tract neurons during postural fixation. J Neurophysiol 32:375-385.

Evarts EV (1981) Role of the motor cortex in voluntary movements in primates. In: Handbook of physiology: the nervous system, Sect 1, Vol II, Pt 2, pp 1083-1120. Bethesda, MD: American Physiological Sociely.

Evarts EV, Fromm C, Kroller J, Jennings VA (1983) Motor cortex control of finely graded forces. J Neurophysiol 49:1199-1215.

Fetz EE, Cheney PD (1978) Muscle fields of primate corticomotoneuronal cells. J Physiol (Paris) 74:239-245.

Fetz EE, Cheney PD (1980) Postspike facilitation of forelimb muscle activity by primate corticomotoneuronal cells. J Neurophysiol 44: $751-772$.

Fetz. FE, Finocchio DV (1975) Correlations between activity of motor cortex cells and arm muscles during operantly conditioned response patterns. Exp Brain Res 3:217-240.

Fisher NI, Lee AJ (1983) Correlation coefficients for random variables on a unit sphere or hypersphere. Biometrika 73:159-164.

Flash T, Hogan N (1985) The coordination of arm movements: an experimentally confirmed mathematical model. J Neurusci 5:16881703.

Fortier PA, Kalaska JF, Smith AM (1989) Cerebellar neuronal activity related to whole-arm reaching movements in the monkey. J Neurophysiol 62:198-211.

Georgopoulos AP, Kalaska JF, Caminiti R, Massey JT (1982) On the relations between the direction of two-dimensional arm movements and cell discharge in primate motor cortex. J Neurosci 2:1527-1537.

Georgopoulos AP, Caminiti R, Kalaska JF, Massey JT (1983) Spatial coding of movement: a hypothesis concerning the coding of movement direction by motor cortical populations. Exp Brain Res (Suppl.) 7:327-336.

Georgopoulos AP, Caminiti R, Kalaska JF (1984a) Static spatial effects in motor cortex and area 5: quantitative relations in two-dimensional space. Exp Brain Res 54:446-454.

Georgopoulos AP, Kalaska JF, Crutcher MD, Caminiti R, Massey JT (1984b) The representation of movement direction in the motor cortex: single cell and population studies. In: Dynamic aspects of neocortical function (Edelman GM, Gall WE, Cowan WM, eds), pp 501-524. New York: Wiley.

Georgopoulos AP, Schwartz AB, Kettner RE (1986) Neuronal population coding of movement direction. Science 233:1416-1419.

Georgopoulos AP, Kettner RE, Schwartz AB (1988) Primate motor cortex and free arm movements to visual targets in three-dimensional space. II. Coding of the direction of movement by a neuronal population. J Neurosci 8:2928-2937.

Gilman S, Carr D, Hollenberg J (1976) Kinematic effects of deafferentation and cerebellar ablation. Brain 99:311-330.

Hamada I, Kubota K (1979) Monkey pyramidal tract neurons and changes of movement parameters in visual tracking. Brain Res Bull 4:249-257.

Hepp-Reymond MC, Wyss UR, Anner R (1978) Neuronal coding of static force in the primate motor cortex. J Physiol (Paris) 74:287291.

Hogan N (1984) An organizing principle for a class of voluntary movements. J Neurosci 4:2745-2754.

Hogan N (1988) Planning and execution of multi-joint movements. Can J Physiol Pharmacol 66:508-517.

Hollerbach JM, Atkeson CG (1987) Deducing planning variables from experimental arm trajectories: pitfalls and possibilities. Biol Cybernet 56:279-292.

Hollerbach .IM, Flash T (1982) Dynamic interactions between limb segments during planar arm movement. Biol Cybernet 44:67-77.

Howell AB, Straus WL (1933) The muscular system. In: The anatomy of the rhesus monkey (Hartman EG, Straus WL, eds), pp 89-175. New York: Hafner.

Humphrey DR, Schmidt EM, Thompson WD (1970) Predicting measures of motor performance from multiple cortical spike train. Science 170:758-762.

Jupp PE, Mardia KV (1980) A general correlation coefficient for directional data and related regression problems. Biometrika 67:163173.

Kalaska JF, Caminiti R, Georgopoulos AP (1983) Cortical mechanisms related to the direction of two-dimensional arm movements: relations in parietal area 5 and comparison with motor cortex. Exp Brain Res 51:247-260.

Kalaska JF, Cohen DAD, Hyde ML, Prud'homme M (1989) A comparison of movement direction-related vs. load direction-related activity in primate motor cortex, using a two-dimensional reaching task. J Neurosci 9:2080-2102.

Kettner RE, Schwartz AB, Georgopoulos AP (1988) Primate motor cortex and free arm movements to visual targets in three-dimensional space. III. Positional gradients and population coding of movement direction from various movement origins. J Neurosci 8:2938-2947.

Lacquaniti F, Soechting JF (1982) Coordination of arm and wrist motion during a reaching task. J Neurosci 2:399-408.

Lemon RN, Mantel GWH, Muir RB (1986) Corticospinal facilitation of hand muscles during voluntary movement in the conscious monkey. J Physiol (Lond) 381:497-527.

Lundberg A (1979) Integration in a propriospinal motor centre controlling the forelimb in the cat. In: Integration in the nervous system (Asanuma H, Wilson VS, eds), pp 47-69. Tokyo: Igaku-Shoin.

Massey JT, Caminiti R, Kalaska JF, Georgopoulos AP (1981) Cortical mechanisms of two dimensional aimed arm movements. VI. Electromyographic analysis. Soc Neurosci Abstr 7:563.

Morasso P (1981) Spatial control of arm movements. Exp Brain Res 42:223-227.

Mountcastle VB, Lynch JC, Georgopoulos A, Sakata H, Acuña C (1975) 
Posterior parietal association cortex of the monkey: command functions for operations within extrapersonal space. J Neurophysiol 38: 871-908.

Murphy JT, Kwan MC, MacKay WA, Wong YC (1982) Precentral unit activity correlated with angular components of a compound arm movement. Brain Res 245:141-145.

Murphy JT, Wong YC, Kwan HC (1985) Sequential activation of neurons in primate motor cortex during unrestrained forelimb movement. J Neurosci 53:435-445.

Mussa-Ivaldi FA (1988) Do neurons in the motor cortex encode movement direction? An alternative hypothesis. Neurosci Lett 91: $106-111$.

Saltzman E (1979) Levels of sensorimotor representation. J Math Psychol 20:91-163.

Schmidt EM, Jost RG, Davis KK (1975) Reexamination of the force relationship of cortical cell discharge patterns with conditioned wrist movements. Brain Res 83:213-223.

Schwartz AB, Ketter RE, Georgopoulos AP (1988) Primate motor cortex and free arm movements to visual targets in three-dimensional space. I. Relations between single cell discharge and direction of movement. J Neurosci 8:2913-2927.

Shinoda Y, Zarzecki P, Asanuma H (1979) Spinal branching of pyramidal tract neurons in the monkey. Fxp Brain Res 34:59-72

Shinoda Y, Yokota JF, Futami T (1981) Divergent projections of individual corticospinal axons to motoneurons of multiple muscles in the monkey. Neurosci Lett 23:7-12.

Smith AM, Hepp-Reymond MC, Wyss UR (1975) Relation of activity in precentral cortical neurons to force and rate of force change during isometric contractions of finger muscles. Exp Brain Res 23:315-332.
Snedecor GW, Cochran WG (1980) Statistical methods, 7th ed. Ames, IA: Iowa State U. P.

Soechting JF, Flanders M (1989a) Sensorimotor representations for pointing to targets in three-dimensional space. J Neurophysiol 62 : $582-594$.

Soechting JF, Flanders M (1989b) Errors in pointing are due to approximations in sensorimotor transformations. J Neurophysiol 62 : $595-608$.

Soechting JF, Lacquaniti F (1981) Invariant characteristics of a pointing movement in man. J Neurosci 1:710-720.

Soechting JF, Terzuolo CA (1988) Sensorimotor transformations underlying the organization of arm movements in three-dimensional space. Can J Physiol Pharmacol 66:502-507.

Stein RB (1982) What muscles variable(s) does the nervous system control in limb movements? Behav Brain Sci 5:535-577.

Steinmetz MA, Motter BC, Duffy CD, Mountcastle VB (1987) Functional properties of parietal visual neurons: radial organization of directionalities within the visual field. J Neurosci 7:177-191.

Thach WT (1978) Correlation of neural discharge with pattern and force of muscular activity, joint positions, and direction of intended next movement in motor cortex and cerebellum. J Neurophysiol 41 : 654-676.

Vogt O, Vogt C, (1919) Allgemeinere Frgebnisse unsererer Hirnforschung. J Psychol Neurol (Leipzig) 25:279-439.

Watson GS (1983) Statistics on spheres. New York: Wiley.

Wiesendanger M (1981) Organization of secondary motor areas of cerebral cortex. In: Handbook of physiology: the nervous system, Sect 1, Vol II, Pt 2, pp 1121-1148. Bethesda, MD: American Physiological Suciely. 